\title{
The Wendelstein Calar Alto Pixellensing Project (WeCAPP): the M 31 variable star catalogue ${ }^{\star, \star \star}$
}

\author{
J. Fliri ${ }^{1, \star \star \star}$, A. Riffeser ${ }^{1,2, \star \star \star}$, S. Seitz ${ }^{1}$, and R. Bender ${ }^{1,2}$ \\ 1 Universitätssternwarte München, Scheinerstrasse 1, 81679 München, Germany \\ e-mail: fliri@usm.uni-muenchen.de \\ 2 Max-Planck-Institut für Extraterrestrische Physik, Giessenbachstrasse, 85748 Garching, Germany
}

Received 21 October 2004 / Accepted 24 August 2005

\begin{abstract}
In this paper we present the WeCAPP catalogue of variable stars found in the bulge of M 31. Observations in the WeCAPP microlensing survey (optical $R$ and $I$ bands) for a period of three years (2000-2003) resulted in a database with unprecedented time coverage for an extragalactic variable star study. We detected 23781 variable sources in a $16.1^{\prime} \times 16.6^{\prime}$ field centered on the nucleus of M 31 . The catalogue of variable stars contains the positions, the periods, and the variation amplitudes in the $R$ and $I$ bands. We classified the variables according to their position in the $R$-band period-amplitude plane. Three groups can be distinguished; while the first two groups can be mainly associated with Cepheid-like variables (population I Cepheids in group I; type II Cepheids and RV Tauri stars in group II), the third one consists of Long Period Variables (LPVs). We detected 37 RV Tauri stars and 11 RV Tauri candidates, which makes this catalogue one of the largest collections of this class of stars to date. The classification scheme is supported by Fourier decomposition of the light curves. Our data shows a correlation of the low-order Fourier coefficients $\Phi_{21}$ with $\Phi_{31}$ for classical Cepheids, as well as for type II Cepheids and RV Tauri stars. Correlating our sample of variable stars with X-ray based catalogues of Kaaret (2002, ApJ, 578, 114) and Kong et al. (2002, ApJ, 577, 738) results in 23 and 31 coincidences, 8 and 12 of which are M 31 globular clusters. The number density of detected variables is clearly not symmetric, which has to be included in the calculations of the expected microlensing event rate towards M 31. This asymmetry is due to the enhanced extinction in the spiral arms superimposed on the bulge of M 31, which reduces the number of sources to about $60 \%$, if compared to areas of equivalent bulge brightness without enhanced extinction present.
\end{abstract}

Key words. galaxies: individual: M 31 - cosmology: dark matter - stars: variables: general - stars: variables: Cepheids - X-rays: stars

\section{Introduction}

In the past decade the microlensing surveys of the galactic bulge and the Magellanic Clouds (LMC and SMC) have greatly extended our knowledge of variable stars. As the observations of these experiments usually cover a long time span with good time sampling, the resulting data sets are perfectly suited for the study of many different types of variable sources. Hence, lots of the progress in this field has resulted from the work of collaborations like MACHO (e.g., Alcock et al. 1995, 1998), EROS (e.g., Beaulieu et al. 1995; Derue et al. 2001), OGLE (e.g., Cieslinski et al. 2003; Wray et al. 2004) or MOA (e.g., Noda et al. 2002; 2004). The list of identified variable

* Based on observations obtained at the Wendelstein Observatory of the Universitätssternwarte München.

$\star \star$ Tables 2-5 and Appendices A, B are only available in electronic form at http://www. edpsciences.org

$\star \star \star$ Visiting astronomer at the German-Spanish Astronomical Center, Calar Alto, operated by the Max-Planck-Institut für Astronomie, Heidelberg, jointly with the Spanish National Commission for Astronomy. stars in the Galaxy and the Magellanic Clouds was enlarged in numerous publications, which not only improved on the number statistics, but has also helped to understand the physical processes dominating these stars better.

M31 has been surveyed for variable sources since the 1920s, starting with the pioneering work of Edwin Hubble. With plates taken at the newly available Mount Wilson telescope, Hubble succeeded in resolving Cepheid variables in the outer parts of M 31. Using the already established periodluminosity relation, Hubble (1922) was able to determine the distance to M31 to $300 \mathrm{kpc}$ (the difference to the actual value of $780 \mathrm{kpc}$ (Stanek \& Garnavich 1998) is mainly due to an erroneous calibration of the zero-point of the PL-relation and a missing reddening correction) and in this way to reveal the extragalactic nature of the "Andromeda Nebula". Baade \& Swope (1963, 1965) continued the work on variable stars in M 31 and detected over 400 variables, among them Cepheids and novae. Looking at the relations between period, luminosity, amplitude, and frequency, they found that these Cepheids resemble the ones in the Milky Way, but seem to be different from those in the Small Magellanic Cloud. More recently, the 
DIRECT project (Kaluzny et al. 1998) performed a comprehensive variable star study in M 31 with the main goal being to reduce the uncertainties in its distance determination. In course of the survey, the DIRECT collaboration identified and examined Cepheids and Detached Eclipsing Binaries in five fields of the M31 disk, which was published in a series of papers. Unfortunately, the data of a 6th field of the bulge of M31, which in part overlaps with our surveyed area, remained unpublished.

During past years Andromeda was a preferred target for a new kind of microlensing experiment. M 31 has the advantage of offering the possibility to distinguish between lensing by dark halo objects (Machos) and lensing by stars in M 31 (a contribution called "self-lensing") in a statistical way, using the near-far asymmetry of the microlensing event rate. As the disk of M 31 is highly inclined $\left(77^{\circ}\right.$, Stanek \& Garnavich 1998) one expects more Macho events towards the far side of the M 31 disk than towards the near side. On the other hand, self-lensing events should not show this signature. Detecting an asymmetry in the event rates would thus help to distinguish between both kinds of events. Several "pixellensing" surveys - AGAPE (Ansari et al. 1997), POINT-AGAPE (Auriere et al. 2001), WeCAPP (Riffeser et al. 2001), MEGA (de Jong et al. 2004), SLOTT/AGAPE (Calchi Novati et al. 2002) - therefore observed the bulge and parts of the disk of M 31 fairly continuously, and also generated catalogues of variable sources as a by-product of these experiments. The work presented in this paper thus overlaps with the work resulting from the AGAPE (Ansari et al. 2004) and POINT-AGAPE (An et al. 2004) data sets.

In contrast to classical microlensing surveys, pixellensing experiments monitor the variation in the surface brightness of a nearby galaxy rather than the variation in the luminosities of resolved stars. Two methods were developed and implemented to overcome the problem of crowding: the super-pixel method (Baillon et al. 1993, used by the AGAPE and POINTAGAPE collaborations) and difference imaging (Tomaney \& Crotts 1996; Alard \& Lupton 1998), which we used to extract and measure the variable sources. The principle of difference imaging is straightforward: positionally and photometrically aligned images of two different epochs are subtracted from one another, and if a source changes its luminosity between the two epochs, it shows up as a point source in the difference image. All blended flux cancels out and contributes only to the noise level in the difference image. The success of difference imaging strongly depends on the quality of the matching of the pointspread-functions (PSFs) between the two images. In our implementation, the residuals in the difference images reach the theoretical Poisson noise limit (see Sect. 2.3). As the Poisson noise of the M 31 surface brightness is the limiting factor for the detection of the variable sources, we stacked all images of one night and generate difference images from these co-added images. Due to this daily stacking scheme, we were not sensitive to variations and to periods smaller than 1.3 days, and focus therefore on longer period variables, namely Cepheids, RV Tauri stars, and Long Period Variables (LPVs), i.e. Miras and semi-regular variables. The catalogue is completed by eclipsing binaries and by variables showing eruptive or irregular variations. The $\delta$ Scuti and RR Lyrae stars show variation amplitudes below our detection limit and will therefore be missed in this study.

The paper is organized as follows. In Sect. 2 we give an overview of the survey, the observations, and data reduction. Section 3 deals with source detection and the derivation of possible periods of the variables. In Sects. 4 and 5, we show the different groups of variable sources detected in the survey. The catalogue of variable stars is presented in Sect. 6 and is correlated with X-ray selected catalogues in Sect. 7. While Sect. 8 summarizes the paper, the appendix deals with the accuracy of the derived periods.

\section{The data set}

\subsection{Description of the survey}

The data presented here result from three years of observing the central part of M 31 by the WeCAPP project. We obtained data from the $0.8 \mathrm{~m}$ telescope at Wendelstein Observatory (Germany) and from the $1.23 \mathrm{~m}$ telescope at Calar Alto Observatory (Spain). At Wendelstein with its field of view (FOV) of $8.3^{\prime} \times 8.3^{\prime}$, we selected a field (F1 in the following) along the minor axis of M 31, which contains the area with the highest expected rate of pixellensing events (see also Fig. 1 for the location of F1). Observations of this field were accompanied by images of F3, the opposite field along the NW minor axis, taken with a sparser time sampling. The Calar Alto field covered $17.2^{\prime} \times 17.2^{\prime}$ centered on the nucleus of M 31. Two quadrants of the field coincided with the maximal lensing field F1 and the opposite field F3. Due to these simultaneous observations, we reached a very good time sampling when M 31 was observable. Since summer 2002 we have been mosaicing fields F1 to F4 solely with the Wendelstein telescope. A composite image ( $V-, R$-, and $I$-band) of fields $\mathrm{F} 1$ to $\mathrm{F} 4$ taken at Calar Alto Observatory during the campaign 2000/2001 is shown in Fig. 1. The epochs with data taken for the four fields are shown in Fig. 2.

\subsection{Instruments and filters}

An overview of the cameras and CCDs used in WeCAPP is shown in Table 1 . The cameras are a $1 \mathrm{~K} \times 1 \mathrm{~K}$ TEK camera with a pixel size of $0.49^{\prime \prime}$ at Wendelstein and a $2 \mathrm{~K} \times 2 \mathrm{~K}$ SITe camera with a pixel size of $0.5^{\prime \prime}$ at Calar Alto. The observations were carried out in $R$ and $I$ filters close to the KronCousins system. At Wendelstein we used the $R(\lambda \simeq 650 \mathrm{~nm}$, $\Delta \lambda \simeq 150 \mathrm{~nm})$ and $I(\lambda \simeq 850 \mathrm{~nm}, \Delta \lambda \simeq 150 \mathrm{~nm})$ wavebands. The Calar Alto observations were carried out with very similar filters, $R(\lambda \simeq 640 \mathrm{~nm}, \Delta \lambda \simeq 160 \mathrm{~nm})$ and $I(\lambda \simeq 850 \mathrm{~nm}$, $\Delta \lambda \simeq 150 \mathrm{~nm}$ ). Despite the combination of different telescopes, CCDs, and slightly different filter systems, we observed no systematic effects in the light curves that depended on these parameters; also see Fig. 24 where we show the data obtained at Calar Alto and at Wendelstein separately. 


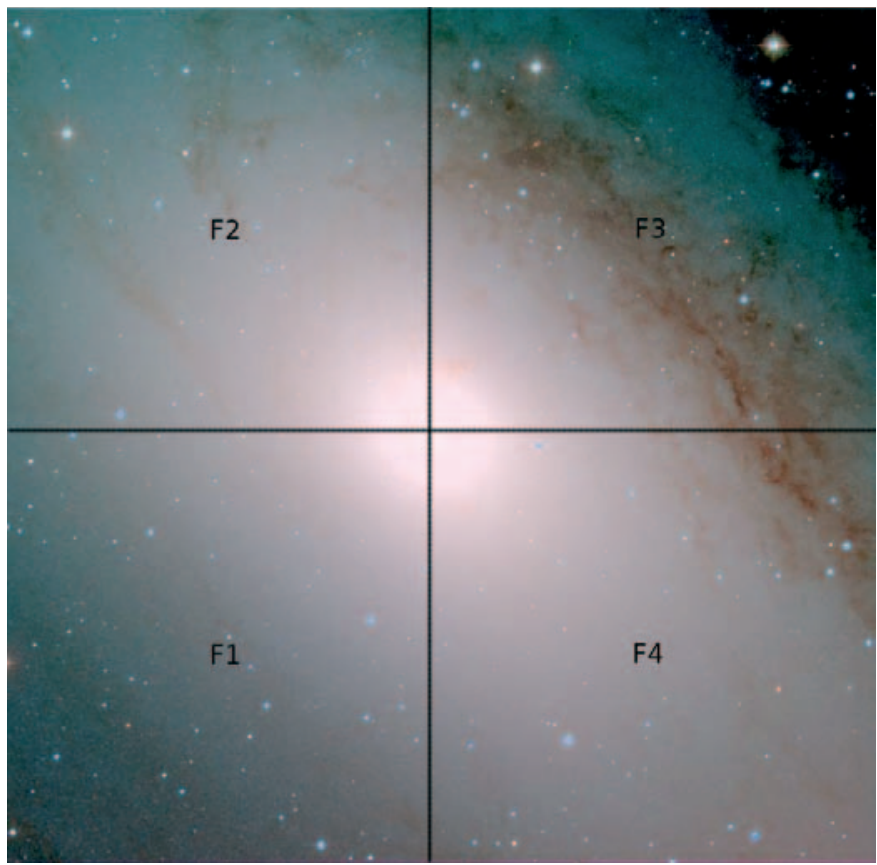

Fig. 1. $V$-, $R$-, and $I$-band composite image of the observed fields F1 to F4, taken at Calar Alto Observatory during the campaign 2000/2001. The black lines mark the positions of fields F1 to F4. The identified variable sources lie within RA(2000): [00h43m25.0s, 00h41m59.9s] and $\operatorname{Dec}(2000)$ : [41d08'00.1', $\left.41 \mathrm{~d} 24^{\prime} 18.0^{\prime \prime}\right]$.

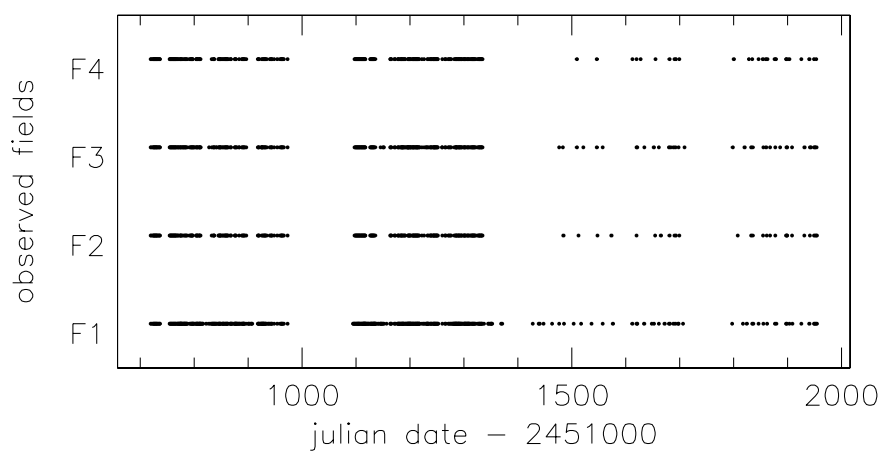

Fig. 2. Distribution of the observations for the four fields. During the first two campaigns, we reached a very dense time sampling of the observations. Note that the third and fourth campaigns were restricted to Wendelstein Observatory only.

\subsection{Data reduction}

The WeCAPP reduction pipeline mupipe will be presented in a future publication (Riffeser et al. 2005, in prep.). It combines all reduction steps from de-biasing of the images until PSF photometry and the final measurements of the light-curve in one software package, including full error propagation from the first reduction step to the last (Gössl \& Riffeser 2002):

1. standard CCD reduction including de-biasing, flatfielding, and filtering of cosmic ray events;

2. position alignment on a reference grid using a flux conserving interpolation routine;

3. photometric alignment of the images;
Table 1. Properties of the CCD cameras used during WeCAPP at Wendelstein (We) and Calar Alto (CA) Observatories. Both CCDs have a pixel size of $24 \mu \mathrm{m}$.

\begin{tabular}{|c|c|c|c|c|c|}
\hline Site & Campaign & $\mathrm{CCD}$ & Size & {$[\operatorname{arcsec} / \mathrm{px}]$} & Field $\left[\operatorname{arcmin}^{2}\right]$ \\
\hline$\overline{\mathrm{We}}$ & $2000-2003$ & TEK \#1 & $1 \mathrm{~K} \times 1 \mathrm{~K}$ & 0.49 & $8.3 \times 8.3$ \\
\hline CA & 2000-2002 & SITe2b \#17 & $2 \mathrm{~K} \times 2 \mathrm{~K}$ & 0.50 & $17.2 \times 17.2$ \\
\hline
\end{tabular}

4. restoration of pixels damaged by CCD defects (cold and hot pixels, traps, bad columns) or pixels hit by cosmic ray events;

5. stacking of the frames of one epoch (i.e. night) using a weighting scheme to maximize the $S / N$ for point sources;

6. matching of the PSF of a high $S / N$ reference frame to the PSF of each stacked frame using our implementation of the image subtraction method developed by Alard \& Lupton (1998) and Alard (2000);

7. generation of difference images by subtracting the convolved reference frame from each stacked frame;

8. PSF photometry of each pixel in the difference frames using a PSF extracted from the convolved high $S / N$ reference frame;

9. extraction of the light curves.

A floating chart of the reduction pipeline is shown in Fig. 3. In the last step, mupipe returns roughly $4 \times 10^{6}$ pixel light curves together with appropriate errors, with each of the light curves representing the time variability of the flux present inside the PSF centered on the particular pixel. The extraction of intrinsic variable sources from these pixel light curves is presented in the next section.

\subsection{Astrometry}

The astrometric solution was created using the $\mathrm{IRAF}^{1}$ tasks ccmap and cctran for 92 stars whose positions were taken from the Local Group Survey (LGS, Massey et al. 2001). The rms of the solution is $0.10^{\prime \prime}$ in declination and $0.12^{\prime \prime}$ in right ascension. The coordinates derived here agree perfectly with the ones derived in Riffeser et al. (2001) and Riffeser et al. (2003).

\section{Detection of variable sources}

\subsection{Selection of the sources}

For the selection of the intrinsic variable sources, we used the $R$-band data from the Calar Alto campaigns 2000/2001 and 2001/2002 to create a $\chi_{v}^{2}$ variation frame. To get rid of systematic effects induced by the different seeing conditions of each frame we used the following approach. We selected a stacked image with roughly the median seeing of the 2000/2001 campaign (about $1.5^{\prime \prime}$ ) as the master frame and matched the PSF of each stacked frame with a smaller PSF to the one of the

${ }^{1}$ IRAF is distributed by the National Optical Astronomy Observatories, which are operated by the Association of Universities for Research in Astronomy, Inc., under cooperative agreement with the National Science Foundation. 


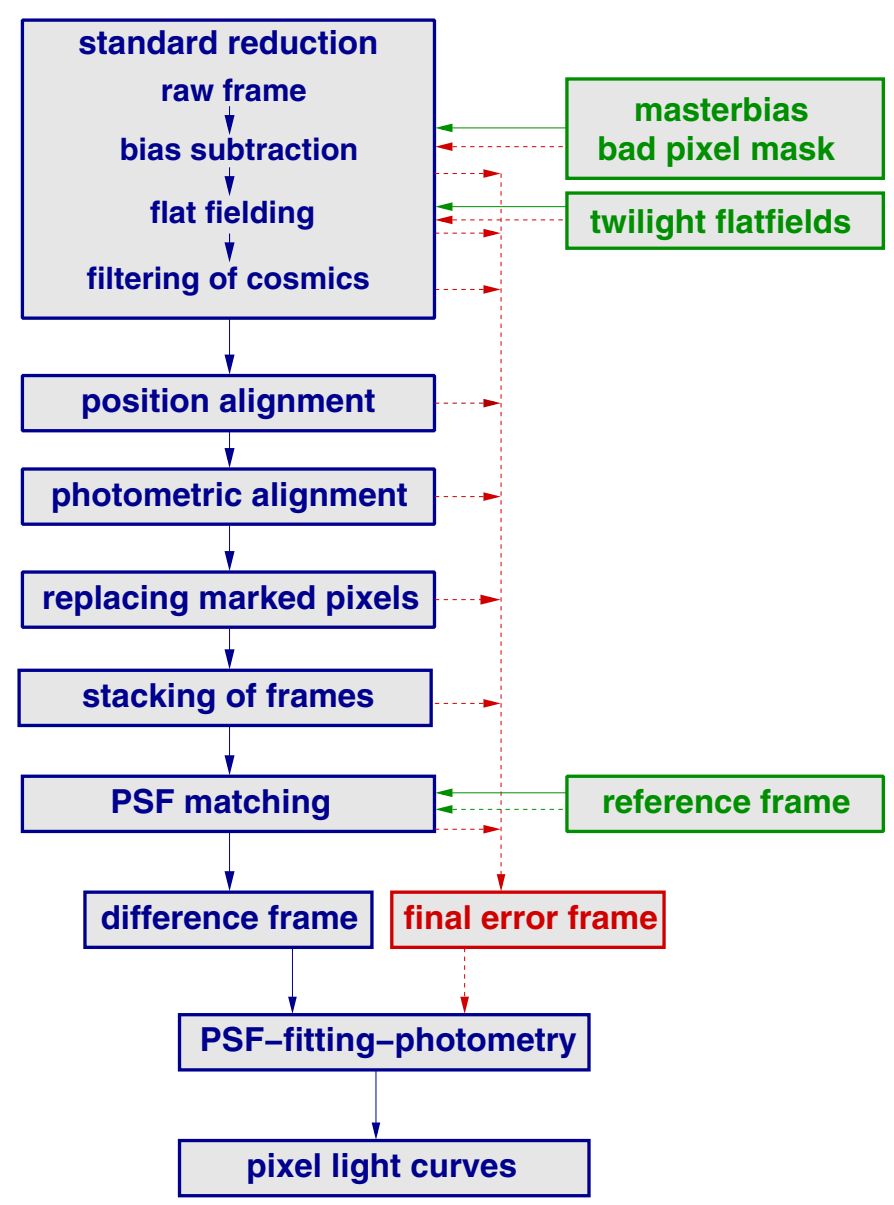

Fig. 3. Floating chart of the WeCAPP reduction pipeline mupipe. The reduction package includes full error propagation for each pixel through all reduction steps. In this way, all data points are properly taken into account in the search for variable sources. The reduction pipeline returns light curves for each pixel in the frame, representing the temporal change of the flux present inside the PSF centered on the particular pixel.

master frame. In this way we ended up with difference images for 113 epochs (i.e. $48 \%$ of the epochs with $R$-band data obtained at Calar Alto), which were then used to detect the variable sources. For each of the light curves extracted from the PSF $_{1.5}$ difference images, we calculated the reduced $\chi_{v}^{2}$ deviation from a constant (i.e. zero) baseline fit. The errors entering these calculations were the errors propagated by mupipe. The results were written into a $\chi_{v}^{2}$-frame of the field. The mode of the $\chi_{v}^{2}$-frame is 1.02 , which shows the accuracy of the propagated errors. Each value $\geq 1.15$ is connected to a non-constant, i.e. variable, source at the $99.99 \%$ confidence level. To avoid contamination of the M 31 sample with foreground objects, we set all pixels in a radius of 5 pixels around the positions of bright foreground stars to zero before detecting the variable sources in the $\chi_{v}^{2}$-frame. We determined the positions of the variable sources using the SExtractor software (Bertin \& Arnouts 1996) for source detection applied on the $\chi_{v}^{2}$-frame. In this way we detected 25571 variable sources in our observed field. Figure 4 shows an extract of the $\chi_{v}^{2}$-frame with the positions of the detected sources marked.

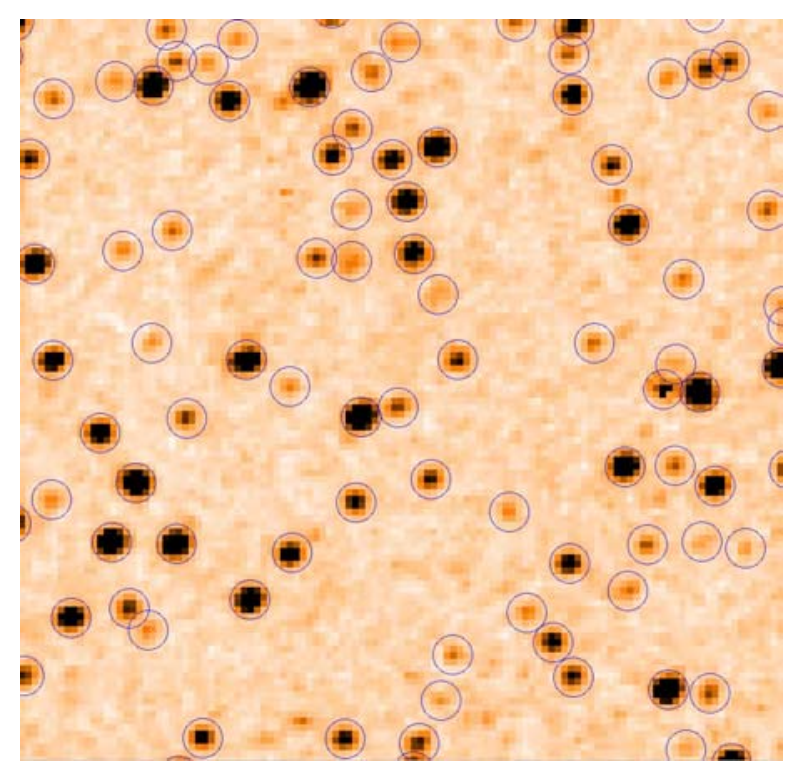

Fig. 4. Extract $\left(1^{\prime} \times 1^{\prime}\right)$ of the $\chi_{v}^{2}$-frame of over 25000 variable sources detected by the WeCAPP microlensing survey of M 31. The circles give the positions of the sources detected by SExtractor. Most of the sources are first identifications, the majority of them being Long Period Variables (LPVs), i.e. Miras and semi-regular variables (see Sect. 4).

\subsection{Period determination}

We used an algorithm developed by Lomb (1976) and Scargle (1982) to determine the significance and value of a possible period of the variables sources. The method of Lomb is wellsuited for this problem as it is able to deal with unevenly sampled data.

The algorithm extracts the power in the first sine and cosine terms for a set of equally spaced frequencies and also yields the significance of the detected peaks in the power spectrum. The Lomb normalized periodogram for $N$ measured data points $h_{j}=h\left(t_{j}\right), j=1, \ldots, N$, taken at epochs $t_{j}$, is defined by

$$
\begin{aligned}
P_{N}(\omega)= & \frac{1}{2 \sigma^{2}} \frac{\left[\sum_{j}\left(h_{j}-\bar{h}\right) \cos \omega\left(t_{j}-\tau\right)\right]^{2}}{\sum_{j} \cos ^{2} \omega\left(t_{j}-\tau\right)} \\
& +\frac{1}{2 \sigma^{2}} \frac{\left[\sum_{j}\left(h_{j}-\bar{h}\right) \sin \omega\left(t_{j}-\tau\right)\right]^{2}}{\sum_{j} \sin ^{2} \omega\left(t_{j}-\tau\right)},
\end{aligned}
$$

where $\omega=2 \pi v=\frac{2 \pi}{P}$ is the angular frequency for period $P$, mean $\bar{h}$, variance $\sigma^{2}$, and constant $\tau$ are defined as follows

$$
\begin{aligned}
& \bar{h} \equiv \frac{1}{N} \sum_{j=1}^{N} h_{j} \\
& \sigma^{2} \equiv \frac{1}{N-1} \sum_{j=1}^{N}\left(h_{j}-\bar{h}\right)^{2} \\
& \tan (2 \omega \tau) \equiv \frac{\sum_{j} \sin 2 \omega t_{j}}{\sum_{j} \cos 2 \omega t_{j}}
\end{aligned}
$$



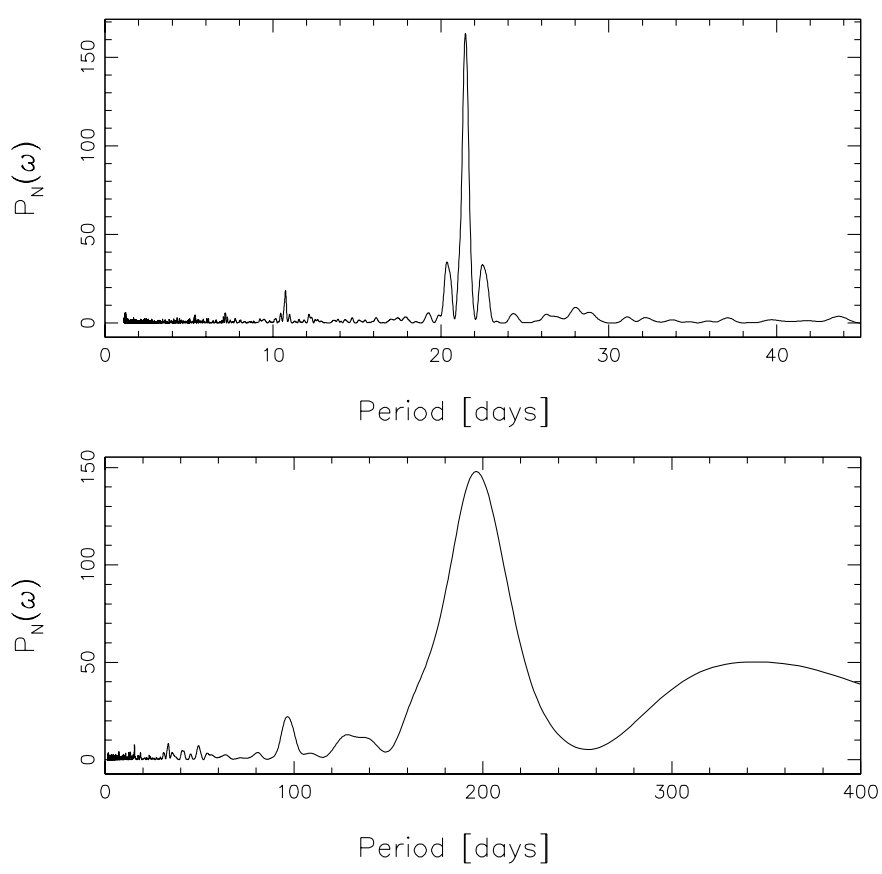

Fig. 5. Two examples of power spectra $P_{N}(\omega)$ of WeCAPP variables. In the upper panel, we show the periodogram for a Cepheid (see also Fig. 20) derived from the $R$-band data; in the lower panel, we show the periodogram for a LPV (see also Fig. 24) derived from the $I$-band data.

The last definition ensures that the power spectrum is independent on a shift of each $t_{j}$ by $\Delta t$, as $\tau$ transforms in that case into $(\tau+\Delta t)$. Lomb (1976) has shown that the evaluation of the periodogram according to Eq. (1) is identical to a linear leastsquares fit of the first harmonics

$F(t)=A \sin \omega t+B \cos \omega t$

to the data points. In Fig. 5 we show two examples for $P_{N}(\omega)$, the first one derived from a Cepheid light curve and the second from the light curve of a LPV.

The significance level $P(>z)$ of a peak with amplitude $z$ in the power spectrum is given by

$P(>z) \equiv 1-\left(1-\mathrm{e}^{-z}\right)^{M}$,

with $\left(1-\mathrm{e}^{-z}\right)^{M}$ being the probability that none of the $M$ tested frequencies shows a greater amplitude than $z$ in case of pure Gaussian noise. Horne \& Baliunas (1986) showed, with extensive Monte Carlo simulations, that the value of $M$, i.e. the number of independent frequencies, does not differ much from the number of data points $N$, if the data points are not closely clumped.

We used the algorithm taken from the Numerical Recipes (Press et al. 1988). Small modifications in the code allowed us to search for the different maxima in the power spectrum. The positions and values of the peaks are returned and can be used for further study of the light curves. In this implementation, the significance $P(>z)$ is approximated for small values of $P(>z)$ by

$P(>z) \approx M \mathrm{e}^{-z}$,

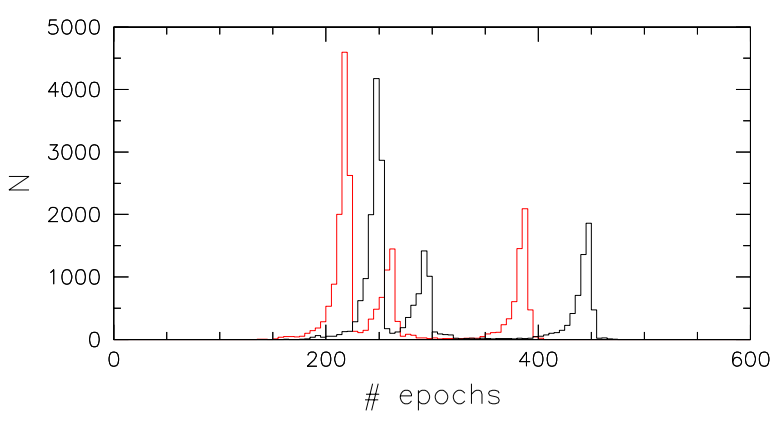

Fig. 6. Histogram of the number of epochs of the level A sources in the $R$-band (black curve) and $I$-band (grey curve, red curve in the online version). For sources located in field F1, we usually got data points in the $R$-band on more than 400 epochs.

where $M$ is a product of the number of data points $N$ and a user supplied value, which determines the high end cut-off of the tested frequencies relative to the Nyquist frequency.

\section{The variable sources}

\subsection{Creating the catalogue}

We detected 25571 variable point sources in a $16.1^{\prime} \times 16.6^{\prime}$ field centered on the nucleus of M31. As this sample still can contain spurious detections or sources with only a few data points measured in the light curve, we applied a couple of cuts which marked different levels of accuracy of the derived periods and showed the need for a visual inspection.

As first cut we demanded that the light curve comprises at least 40 data points in both filters. All sources which did not pass this criterion we removed from the sample. This reduced the number of variables under inspection to 25316 and defined the level A sample. In Fig. 6 we show the histogram of the number of epochs for these sources in the $R$-band (black curve) and $I$-band (red curve). The lowest number peaks represent the time sampling present in fields F2 and F4, followed by the peak corresponding to field F3. As F2 and F4 were observed with comparable frequency, both fields contributed to the lowest number peak. The last peak, with usually more than 400 epochs in the $R$-band, is related to field F1.

As the Lomb algorithm assumes that all data points have the same error (see Eqs. (1) and (3)), data points with big error bars or outliers can spoil the period-finding process and yield spurious periods. To avoid this, we first eliminated in each light curve ten data points with the biggest errors and, in a second step, five data points with the highest as well as five data points with the lowest values. In doing so, we ensure that we have at least 20 data points in both filters to look for a periodic signal.

We then checked the $R$-band and $I$-band light curves separately for periodicities. As the color of variable stars usually changes during a cycle, combing the two data sets would not result in accurate determinations of the period of the sources. Finally we obtained 25316 variables with determined periods (of any significance level) in both bands.

We regard those periods as real that are the same in both bands inside tight boundaries reflecting the error of the period determination. For periods $P_{R}<215$ days, we took the 
theoretical error (see Eq. (A.2)) resulting from $v_{F W H M}$ as limit, while from 215 days onwards we chose a more conservative constant limit of 30 days, which was increased to 60 days for $P_{R}>400$ days. This criterion defined the level $B$ sample (20311 objects).

As the final cut we selected all those variables from level B which showed a significance level of the period determination of $P(>z)<10^{-10}$ in $R$ or $I$ and which had a determined $R$-band period $P_{R} \leq 450$ days. This final cut reduced the number of sources to 19551 and defined the level $C$ sample with welldetermined periods. The remaining sources in level B were inspected visually and assigned to the group of regular, irregular, long-variation (period could not be determined because of an incomplete cycle) or miscellaneous variables in the final catalogue, or were rejected from the sample as spurious detections. Finally, all sources of the level C sample were added automatically to the final catalogue.

For the variables which showed deviant periods in $R$ and $I$, we proceeded as follows. If the significance level of one of the deviant periods was better than $10^{-15}$ and at the same time better than the significance in the other band by a factor of $10^{10}$, we chose the period in the first band and add the variable to the final catalogue. If this was not the case, we inspected the light curve visually and decided if one of the periods was the real one, or if the variation was of an irregular nature. In this step we also rejected the last spurious detections from the sample and the true variables were added to the final catalogue.

\subsubsection{Search for eclipsing binaries}

By visual inspection of the folded light curves of the Lomb sample, we detected 28 eclipsing binary (EB) candidates, amongst them one (semi-)detached system. However, the Lomb algorithm can fail in detecting periods for potential EB systems in M 31. As EB light curves, especially the ones of detached systems, show strong power in the higher harmonics, they are not recovered well by Fourier techniques using only the first harmonic. Since some EB candidates could therefore be missed by using the Lomb algorithm alone, we added another step to the search for EBs. We once more investigated all light curves that had been removed in the previous steps; however this time we used the dedicated transit finding algorithm "boxfitting" (Kovacs et al. 2002) for the period determination. As Tingley (2003) has shown, the boxfitting algorithm is a powerful tool for detection of eclipsing systems. We implemented the original Fortran code ${ }^{2}$ into our detection pipeline and ran the algorithm over the mostly noisy light curves that had been removed from the sample as spurious detections in the previous steps. In this way we made certain to have proper periods for all potential EB candidates present in our variable sources.

Selecting all light curves with a reasonable signal detection efficiency (SDE, Kovacs et al. 2002) SDE $>$ 6, periods $P>1.30$ days and rejecting periods between 1.95 days and 2.05 days (to avoid to pick up aliasing periods) together resulted in an additional sample of 155 light curves. Visual

\footnotetext{
${ }^{2}$ http://www.konkoly.hu/staff/kovacs.html
}
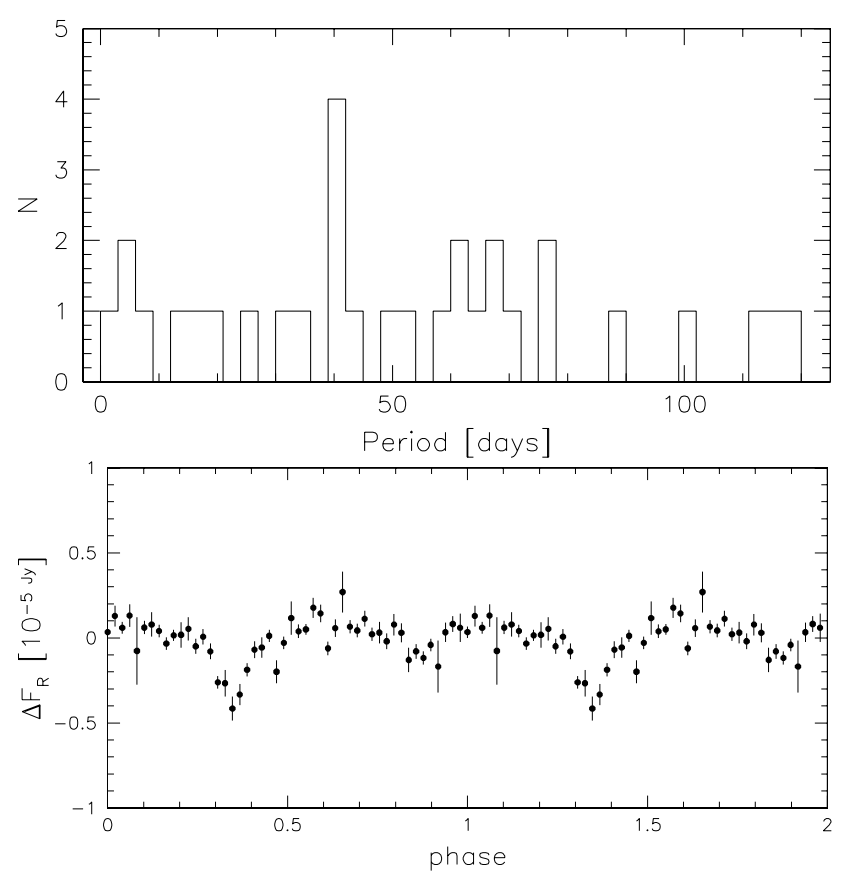

Fig. 7. Top panel: histogram of the periods of the 31 eclipsing binary candidates. The 4 (semi-) detached systems populate the low period area up to a period of 7 days, whereas the candidate contact systems generally have longer periods. Bottom panel: binned $R$-band light curve of a semi-detached system in the phase representation. The system has a $R$-band period of 7.0791 days.

inspection of the folded light curves finally yielded 3 additional candidates for (semi-) detached eclipsing binary systems.

In Fig. 7 we show the distribution of periods of the $31 \mathrm{~EB}$ candidates in the upper panel. The 4 (semi-) detached systems populate the low period area up to a period of 7 days, whereas the candidate contact systems generally have longer periods. In the bottom panel, we show the binned $R$-band light curve of a semi-detached system in the phase representation. The system was detected using the Lomb algorithm and has a $R$-band period of 7.0791 days.

\subsection{Number counts and asymmetry}

The positions of the variables from the final catalogue suggest a connection of the enhanced extinction in M 31's spiral arms and the depletion of sources in certain regions in the northern part of the bulge (field F3). This depletion is also evident from number counts of the sources in the northern and southern hemispheres of M 31 (see Fig. 8).

By summing up all variables in $100 \times 100$ pixel [ $50^{\prime \prime} \times 50^{\prime \prime}$ ] bins, we calculated the number density of variable sources. In the resulting density map (Fig. 9), the spiral arms are clearly visible. A comparison of the number densities at equivalent positions in the M 31 bulge - one in the dust lane of the spiral arms, the other in the opposite hemisphere - shows a reduction to about $60 \%$ compared to the part with no strong extinction.

To examine this subject further, we compared the number density map (see Fig. 9) with an extinction map of our field. In Fig. 10 we plot the positions of the sources on top of the 


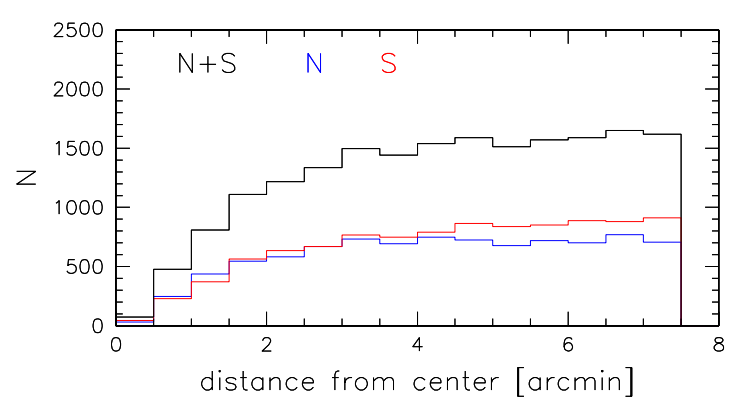

Fig. 8. Number counts of sources of the catalogue as function of the distance to the center of M 31. Black: all sources. Blue: sources in the northern hemisphere of M 31. Red: sources in the southern hemisphere of M 31. The asymmetry of the detected sources is clearly visible and is due to the enhanced disk-extinction in the northern part. The low number of sources near the center is due to incompleteness induced by crowding and saturated parts in the frames.

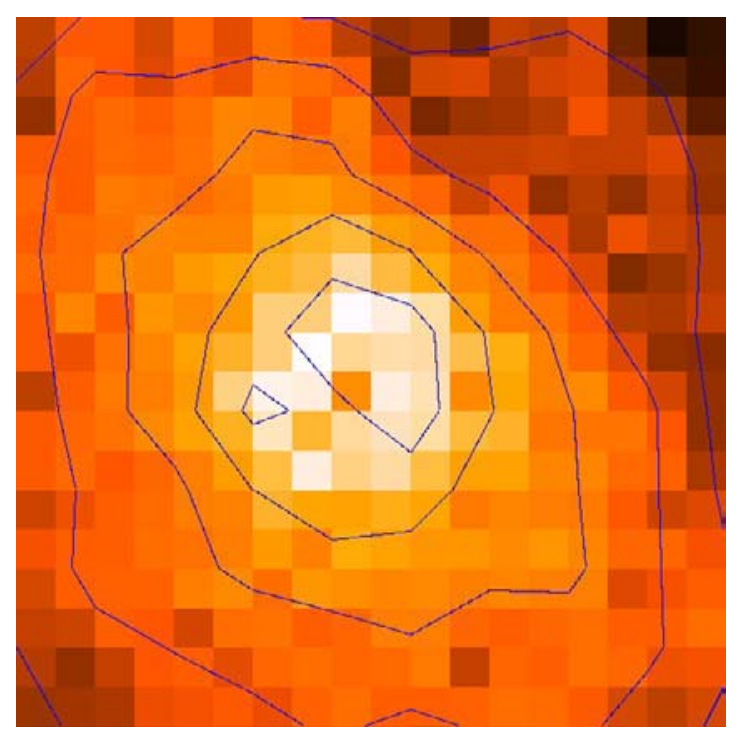

Fig. 9. Number density of variable sources by summing up all variables in $100 \times 100$ pixel $\left[50^{\prime \prime} \times 50^{\prime \prime}\right]$ bins. To avoid effects induced by the border of the frame, we only use pixels in the region [200:2000, 200:2000]. The contour levels are 1, 2, 3, 4, and $5 \times 10^{-2}\left[\operatorname{arcsec}^{-2}\right]$, respectively. The spiral arm is clearly visible. Due to extinction in the dust lanes, the number density reduces to about $60 \%$ when compared to equivalent regions without strong dust features present. The regions with underdensities near the center are due to saturated parts in the frame with no variable sources detected.

$R$-band extinction map, which we derived from the $V$ - and $R$ band frames taken by the LGS survey in the following way:

We started from the relation between the color excess $E(V-$ $R$ ) and the extinction $A_{R}$ in the $R$-band ( $a \approx 3$, see Binney \& Merrifield 1988)

$A_{R}=a E(V-R)$.

With the non-reddened magnitudes $M_{R, 0}, M_{V, 0}$ and reddened counterparts $M_{R, r}, M_{V, r}$, this writes as

$M_{R, r}-M_{R, 0}=a\left[\left(M_{V, r}-M_{R, r}\right)-\left(M_{V, 0}-M_{R, 0}\right)\right]$.

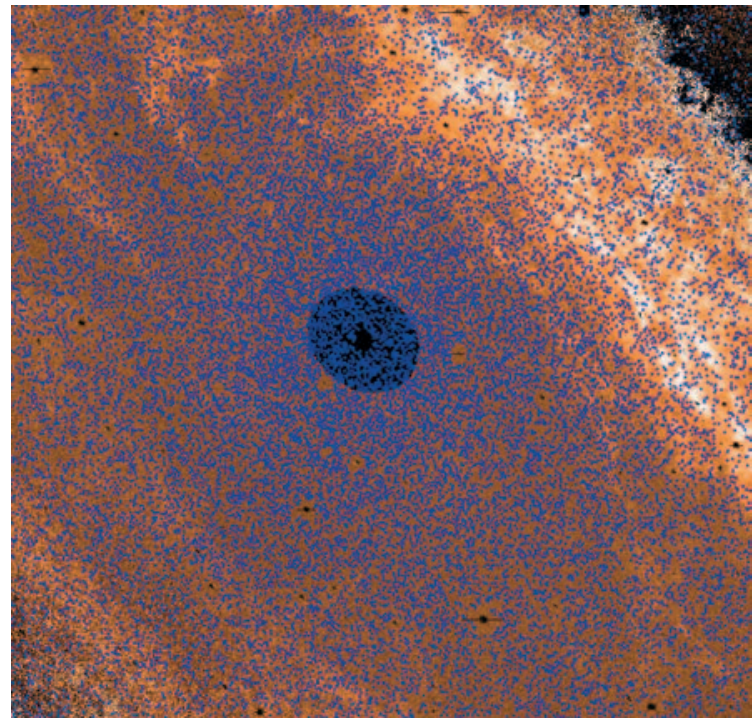

Fig. 10. $R$-band extinction map of the M 31 field. White areas correspond to high extinction values. The extinction in the $R$-band was derived using the $V$ - and $R$-band images taken from the LGS survey (Massey et al. 2001). Overplotted are the positions of the catalogue variables (blue dots). In the central regions, the extinction map could not be calculated due to saturation in the original LGS frames.

The transformation to fluxes $F_{i, j}(i=R, V ; j=0, r)$ yields

$F_{R, 0}=F_{R, r}\left(\frac{F_{R, r}}{F_{V, r}}\right)^{a}\left(\frac{F_{V, 0}}{F_{R, 0}}\right)^{a}$.

If we now suppose that the intrinsic stellar population gradients are negligible over the field, which is not exactly true but a valid approximation for our purposes, we can set $\left(\frac{F_{V, 0}}{F_{R, 0}}\right) \approx$ const.

Using this assumption, we finally obtain a relation for $F_{R, 0}$

$F_{R, 0}=\frac{F_{R, r}^{1+a}}{F_{V, r}^{a}}\left(\frac{F_{V, 0}}{F_{R, 0}}\right)^{a}$,

which can be used to calculate the extinction $\operatorname{ext}_{R}$ in the $R$-band

$\operatorname{ext}_{R}=-2.5 \log \left(\frac{F_{R, r}}{F_{R, 0}}\right)$.

For the intrinsic color $(V-R)_{0}$ required in the calculation we use the theoretical value $(V-R)_{0}=0.63$, assuming the bulge to be a 12 Gyr old SSP of $2 Z_{\odot}$ metallicity (C. Maraston, priv. comm., based on Maraston 1998). Figure 10 shows the resulting extinction map, indeed, under-dense variable source regions coincide with high extinction regions.

Finally we compared the number densities of sources present in our catalogue with theoretical predictions. According to Renzini (1998), the number of LPVs per integrated $10^{5}$ bolometric luminosities is equal to 0.5 . Renzini (1998) uses a slightly older ( $15 \mathrm{Gyr})$ and less metal-rich $\left(1 Z_{\odot}\right)$ bulge when compared to the model used for calculating the extinction frame. With the small bulge decomposition of Kent (1989) and under the assumption that the LPVs are present in the bulge only, we derived the expected number densities of LPVs and show it in Fig. 11. Agreement with the detected number densities is good in the outer parts of the field. Towards 


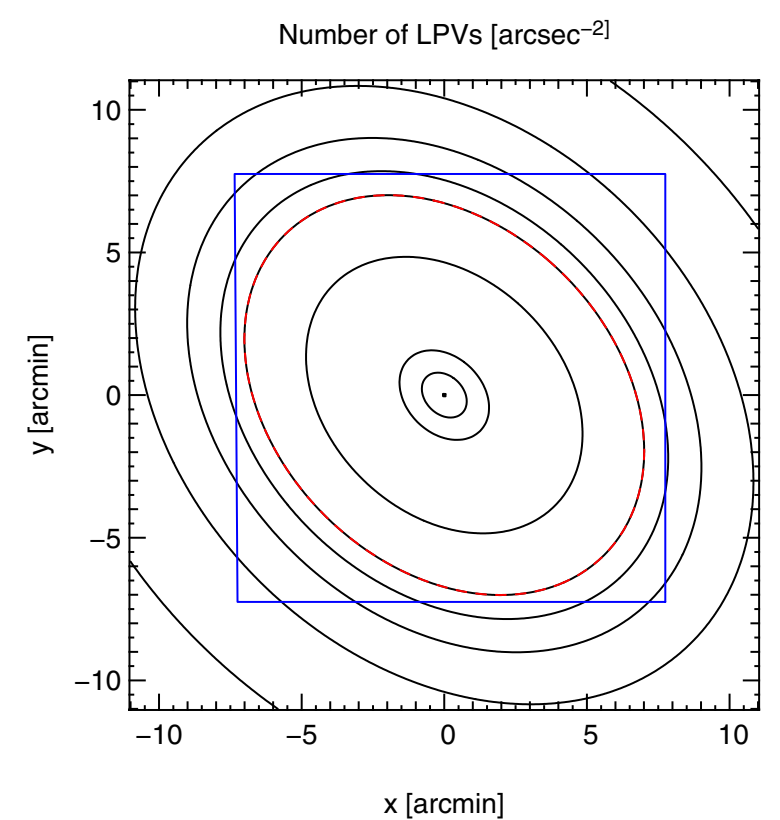

Fig. 11. Theoretically expected number densities of LPVs according to Renzini (1998) calculated with the small bulge decomposition of Kent (1989). The field is centered on the nucleus of M 31; North is on top, and East is on the left of the diagram. The WeCAPP field for which we calculated the number density in Fig. 9 is shown by the grey rectangle (blue rectangle in the online version). Contour levels are $1,2,3,4$ and $5 \times 10^{-2}, 0.1,0.5,1,5$ and 10 sources [ $\operatorname{arcsec}^{-2}$ ]. The black-grey dashed line (red line in the online version) marks the $5 \times 10^{-2}$ contour level.

the center, we suffer from incompleteness due to enhanced noise, on the one hand, and crowding of the sources, on the other.

\subsection{Classification scheme}

As practically all of the detected sources are unresolved in the original frames, we cannot derive luminosities for the variable sources. Therefore, we are neither able to put the sources in the color-magnitude plane and to construct a color-magnitude diagram nor to derive period-luminosity relations for the different classes. The parameters available for establishing a classification scheme are therefore reduced, leaving the period, its significance, the amplitude of the variation, its (flux excess)color, and finally the light curve shape as classification parameters. The light curve shape is of particular interest as it can be described mathematically and parameterized in terms of the parameters resulting from low order Fourier fits to the data. For classical Cepheids, the Fourier parameters show a progression with the period of the variation, echoing the well-known "Hertzsprung progression" (Hertzsprug 1924) of the light curve shape with period. For type II Cepheids similar correlations have already been found; analysis of the RV Tauri stars in our sample reveals a correlation between different phase parameters. Fourier decomposition of the light curves is, therefore, a powerful method to support the classification of Cepheid-like variables and distinguish them from other types of variables. Our classification scheme is based on the position of the stars in

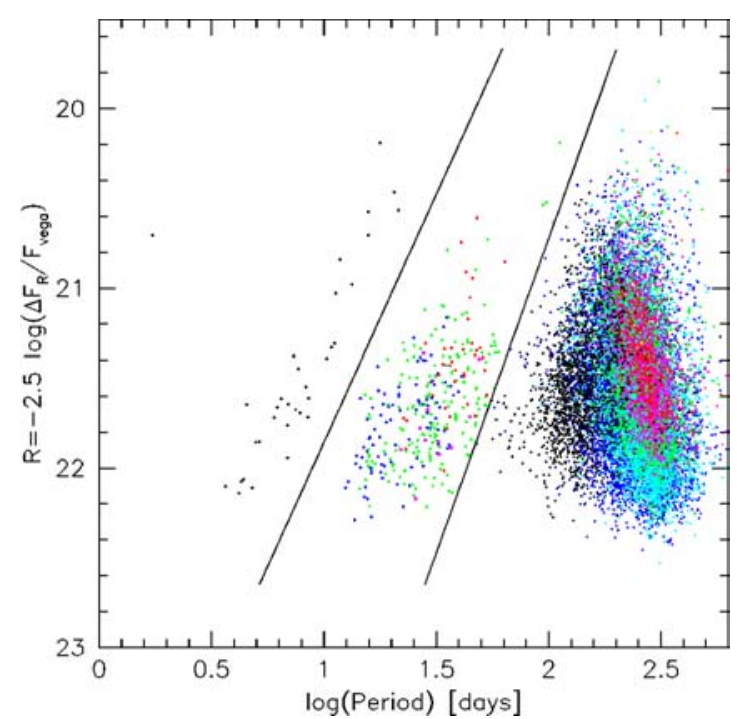

Fig. 12. Period-amplitude relation in the $R$-band for the catalogue sources (eclipsing binaries, irregular, long-variation, and miscellaneous variables excluded). The amplitudes were transformed into magnitudes using the $R$-band fluxes of Vega. Note that these magnitudes only reflect the flux difference and are not the real variation magnitude of a single star. Three populations are visible in this diagram. The black lines show the defining relations (from left to right) for group I (population I Cepheids: black dots), group II (type II Cepheids: blue dots; RV Tauri stars: red dots; RV Tauri candidates: open magenta circles; SR variables: green dots), and group III (LPVs). For the RV Tauri stars and candidates, we use the single or fundamental period (minimum to minimum). The sources in group III are colorcoded according to the significance of the $I$-band period as returned by the Lomb algorithm (low significance: black $\rightarrow$ blue $\rightarrow$ red: high significance).

the $R$-band period-amplitude plane. For certain groups of stars, we refined and checked our classification by using the Fourier parameters of the fits to the light curves.

\subsubsection{Period-amplitude-relations}

Amplitudes $\Delta F_{R}$ and $\Delta F_{I}$ of the variation in the $R$-band and $I$ band are determined as half the difference of the maximum to the minimum of the light curves. As we calculated the amplitudes after having eliminated data points with the largest error bars and biggest flux differences, these amplitudes act only as a lower limit, but can be regarded as a robust measurement of the variation amplitude. The amplitudes were transformed in magnitudes using the $R$ - and $I$-band fluxes of Vega taken from Binney \& Merrifield (1988). Note that these magnitudes reflect the flux difference on the frame only, and are not the real variation magnitudes of a single star.

Figure 12 shows the relation between the logarithm of the period and the logarithm of the amplitude $\Delta F_{R}$ (i.e. the variation magnitude as measured in the frame) for the sources of the final catalogue. Three different groups can be distinguished in this diagram:

- Group I has periods between 1.7 and 21.5 days, and there seems to be a correlation of the period and the amplitude of the variation as bigger periods show larger amplitudes. We 
required $-2.5 \log \left(\Delta F_{R} / F_{\text {Vega }}\right)<23.1-2.76\left(\log P_{R}-0.55\right)$ for sources belonging to this group. This relation, as well as the dividing relation for group II sources, is shown as a black line in Fig. 12. These stars are most likely connected to the disk, as their light curves and the period regime are connected to Cepheids of type I which belong to a young population.

- Group II has periods in the range 12 and about 140 days. Also in this group, the period and the variation amplitude are correlated, whereas the sequence for the group II stars lies at fainter magnitudes than the one for the group I stars. The defining relations for this group are given by $-2.5 \log \left(\Delta F_{R} / F_{\text {Vega }}\right)>23.1-2.76\left(\log P_{R}-0.55\right)$ and $-2.5 \log \left(\Delta F_{R} / F_{\text {Vega }}\right)<23.0-3.5\left(\log P_{R}-1.35\right)$. Group II stars most likely belong to the old spheroid population; RV Tauri stars, type II Cepheids, and the low period tail of the semi-regular (SR) stars are found in this group.

- The bulk of the detected variables finally belongs to group III, variables with periods longer than about 50 days. For this group no clear correlation between the amplitude and the period can be seen. All sources with $-2.5 \log \left(\Delta F_{R} / F_{\text {Vega }}\right)>23.0-3.5\left(\log P_{R}-1.35\right)$ belong here.

\subsubsection{Fourier fits}

We fitted truncated Fourier series of the form

$C+A_{0} \Sigma_{i=1}^{N} A_{i} \cos \left(i \omega\left(t-t_{0}\right)+\Phi_{i}\right)$

to the $R$-band light curves of groups I and II. Here $C$ defines the baseline of the fit, $A_{0}$ reflects the overall amplitude of the light curve, and $A_{i}$ and $\Phi_{i}$ define the amplitudes and phases of the different harmonics. The periods entering the fit are those returned by the Lomb algorithm. A fitting order of $N=5$ leads to acceptable fits to the data.

Simon \& Lee (1981) were the first to calculate amplitude ratios of the form $R_{i j}=A_{i} / A_{j}$ and phase differences $\Phi_{i j}=\Phi_{i}-i \Phi_{j}$ of the parameters of the different harmonics. Since for classical Cepheids both definitions show a progression with the period, as well as a correlation among one another, Fourier analysis proved to be an excellent diagnostic for examining the pulsation properties of these stars. Fourier analysis therefore was widely used in the past to examine Cepheids (e.g., Simon \& Moffett 1986; Alcock et al. 1999), particularly to distinguish between fundamental and first overtone pulsators (e.g., Antonello \& Poretti 1986; Beaulieu et al. 1995).

\section{Classes of variables}

\subsection{Group I and II - Cepheid-like variables}

Groups I and II are populated by Cepheid-like variables (population I Cepheids in group I, type II Cepheids and RV Tauri stars in group II) and by the small period tail of semi-regular variable stars, which start to contribute at periods from about 16 days onwards.

\subsubsection{Population I Cepheids}

Classical Cepheids are relatively young, intermediate mass population I stars. They pulsate in the fundamental mode, which discriminates them from s-Cepheids, that are believed to pulsate in the first overtone. Both groups of population I stars have distinctive light curves, with the classical showing skewed and the s-type showing smooth sinusoidal variations.

We detected 33 population I Cepheids in our sample. To check whether our classification is correct and if there are first overtone pulsators (s-Cepheids) amongst the detected Cepheids, we fitted truncated Fourier series (see Sect. 4.3.2) to the light curves of group I. Figure 13 shows the characteristic progression of the Fourier parameters with the period of the light curve for the classical Cepheids: as the amplitude ratio $R_{21}$ between the first and second harmonic drops the corresponding phase difference shows a mild rise. The amplitude ratio $R_{21}$ declines until a period of about 10 days is reached, and then starts to rise again. Due to suppression of the second harmonic, the Cepheid light curves in the vicinity of this period look quite sinusoidal. Figure 13 shows that the minimum of $R_{21}$ is connected to a dramatic change of $\Phi_{21}$. It is generally believed that this change is connected to the resonance between the fundamental mode and the second overtone $P_{2} / P_{0} \approx 0.5$ (e.g., Simon \& Moffett 1986).

First overtone pulsators can be detected using the amplitude ratio $R_{21}$, as s-Cepheids show lower values than their fundamental mode counterparts. Following Beaulieu et al. (1995) we demanded $R_{21}<0.3$ for $P<3$ days and $R_{21}<0.2$ for $3 \leq P<5.5$ days for the Cepheids to be classified as first overtone pulsators. Using this criterion we identified 2 first overtone pulsators in our sample. One source with a $R_{21}$ value close to the border with the s-Cepheids fell off the fundamental mode sequence in the phase difference diagram. Following Beaulieu et al. (1995), we classify this source as an intermediate Cepheid.

One source is clearly separated from the Cepheid relation in the period-amplitude plane (see Fig. 12). This variable shows Cepheid-like variations that are modulated by a period of 208 days in $R$. The modulation is most likely due to another variable source inside the PSF. Fourier decomposition of the $R$-band data shows that the source has two excited periods, 1.7330 days and 2.3515 days, besides the subdominant modulation of the light curve. In Fig. 14 we show the power spectrum of this source. The two periods would classify the source as a beat Cepheid, the first one detected in $M$ 31. Beat Cepheids are a rare sub-class of Cepheids in which two pulsation modes are excited simultaneously. The ratio of the two periods $P_{\text {short }} / P_{\text {long }} \approx 0.737$ makes it likely that we see a fundamental mode / first overtone $(\mathrm{F} / 1 \mathrm{H})$ pulsator, if the Cepheid hypothesis for this source is confirmed. For $\mathrm{F} / 1 \mathrm{H}$ beat Cepheids, the first overtone should be the dominant mode, which is fulfilled for our candidate. Interestingly, the position in the $\left(P_{1} / P_{0}\right)-\log \left(P_{0}\right)$ diagram would place this beat Cepheid on a sequence defined by the SMC beat Cepheids of Beaulieu et al. (1997), well above the relations for the LMC and the Galaxy. This would point to a Cepheid in M 31 of approximately the same metal content by mass as the SMC beat 

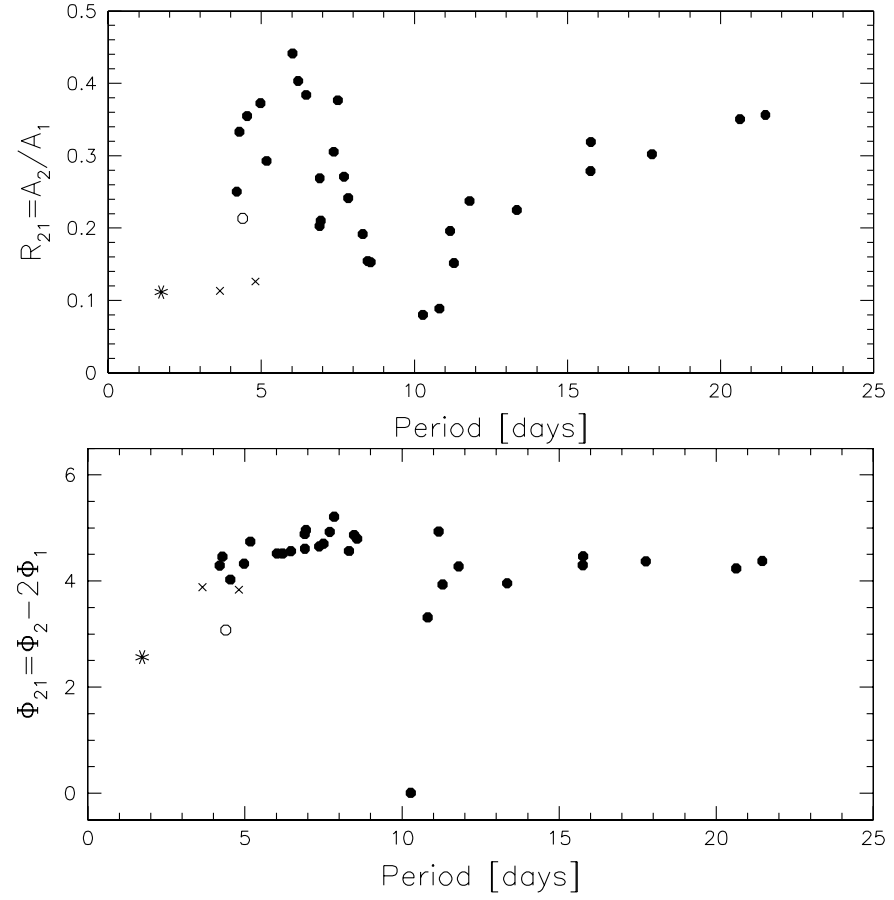

Fig. 13. Amplitude ratio $R_{21}=A_{2} / A_{1}$ (upper panel) and phase difference $\Phi_{21}=\Phi_{2}-2 \Phi_{1}$ (bottom panel) determined from the $R$-band data for the population I Cepheids (group I) plotted against the period. The ratio and phase difference drop as the periods approach the resonance $P_{2} / P_{0} \approx 0.5$ at about 10 days and rise again afterwards. The $R_{21}$ value classifies 29 of the detected Cepheids as fundamental mode pulsators (closed circles), whereas 2 are overtone pulsators (crosses). One of the Cepheids falls off the fundamental mode sequence in the phase difference diagram. Following Beaulieu et al. (1995), we classify this source as intermediate type Cepheid (open circle). The beat Cepheid candidate for which we used the dominant period (presumably the first overtone) in the analysis is marked with an asterisk.

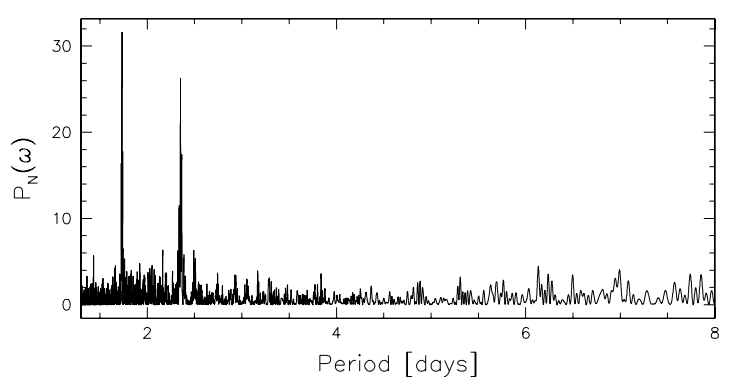

Fig. 14. Power spectrum of the beat Cepheid candidate in the $R$-band. The two peaks in the spectrum correspond to periods of 1.7330 days and 2.3515 days.

Cepheids. The position of this object coincides with a resolved source in our reference image, one which is classified as a star (261 262) in the Haiman et al. (1994) catalogue and which also correlates with an X-ray source in the Kong et al. (2002) catalogue. As the X-ray hardness ratio of this source points to a soft spectrum consistent with stellar X-ray emission, it is classified as a possible foreground star in the Kong et al. (2002) catalogue (J004301.8+411726, see also Table 4). Future investigations will show if these correlations are real or coincidental.
In addition to the beat Cepheid, there is another peculiar source in group I that remains unclassified at this stage. It shows two periods of 6.9489 and 20.4960 days. We will investigate that object further, together with the beat Cepheid candidate, and also present a more detailed description of the Fourier parameters of group I stars in a future publication.

\subsubsection{Type II Cepheids and RV Tauri stars}

Type II Cepheids are low-mass population II variables that follow a period luminosity relation about $1.5 \mathrm{mag}$ below the classical Cepheid relation. They are found in old populations like globular clusters, the halo, or the bulge. Type II Cepheids with periods less than 5 days are also called BL Her or CVB stars, whereas type II Cepheids with periods between 10 and 20 days are often referred to as W Virgini stars. At the upper period limit, a period doubling can often be observed in the light curves. An approximate period limit of about 20 days Alcock et al. (1998) separates type II Cepheids from RV Tauri stars that share the same light curve and often also the same chemical and dynamical characteristics (Fokin 2001). RV Tauri stars can be recognized by a typical double-wave light curve with alternating deep and shallow minima, their semi-periods (minimum to minimum) ranging from 20 to over 50 days. The current understanding places RV Tauri stars at the end of stellar evolution. After leaving the Asymptotic Giant Branch (AGB, Iben \& Renzini 1983), they move left in the HR-diagram to enter the instability strip at high luminosities. For a recent review of population II Cepheids and related stars see Wallerstein (2002). A comprehensive collection of type II Cepheids and RV Tauri stars detected in the LMC by the MACHO collaboration can be found in Alcock et al. (1998).

We detected 37 RV Tauri stars and 11 RV Tauri candidates, which makes this catalogue one of the biggest collections of RV Tauris to date. The light curves show the typical alternation of deep and shallow minima, the second maximum being fainter than the first one, although for a few sources this latter difference is rather marginal. The light curve shape can be divided into two groups, one resembling the "flat-topped" Cepheid II shape (see Fig. 22), the other showing sinusoidal variations. To avoid misclassification of the sinusoidal light curves of RV Tauri stars with the not-too-different light curves of $\beta$-Lyrae eclipsing binaries, we extracted the phase difference between the second and the fourth harmonic from Fourier fits to the light curves. According to Szymanski et al. (2001) these two phases should be strongly coupled for sinusoidal contact systems, yielding $\Delta \Phi=\Phi_{4}-2 \Phi_{2}=0$ (M. Szymanski, priv. comm.). For pulsating stars, the correlation is much weaker. Five of our RV Tauri candidates with sinusoidal light curves have a $\Delta \Phi$ within $1-\sigma$ compatible with 0 (or equivalently $2 \pi$ ) and another 6 lie within 3- $\sigma$. We classified all $11 \mathrm{RV}$ Tauris whose $\Delta \Phi$ values are within 3- $\sigma$ compatible with 0 as RV Tauri candidates, and the remaining 37 sources as RV Tauri stars.

Using the fundamental period (minimum to minimum) for the Fourier extension of the RV Tauri sources yields relatively bad fits and subsequently more uncertain Fourier parameters. We therefore used the formal period in the analysis, which 


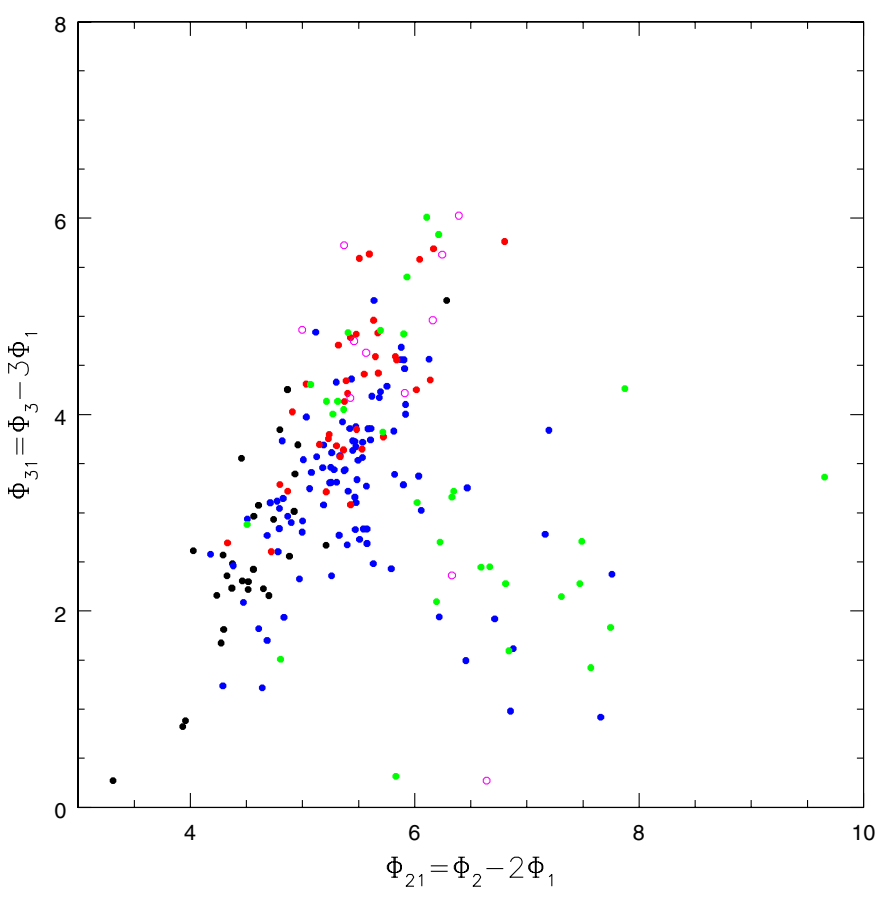

Fig. 15. Phase differences $\Phi_{i j}=\Phi_{i}-i \Phi_{j}$ as determined from the $R$ band data plotted against each other. Black dots: population I Cepheids (group I); blue dots: type II Cepheids (group II); red dots: RV Tauri stars (group II); open magenta circles: RV Tauri candidates (group II). As green dots, we show the LMC type II Cepheids and RV Tauri stars from Alcock et al. (1998), which fall on the sequences of the WeCAPP sources. For the RV Tauri stars, we use the formal period in the analysis. The classical Cepheids show a clear correlation for the two parameters, but also for the type II Cepheids and the RV Tauri stars both phase differences are correlated. The sequences of RV Tauri stars and type II Cepheids overlap, making the RV Tauri sequence into an extension of the Cepheid II sequence. This favors the close connection between these two types of stars.

yields a more appropriate description of the data, better fits, and more reliable Fourier parameters. In Fig. 15 we show a "phase-phase" diagram as a further result of the Fourier analysis of the RV Tauri and candidate RV Tauri light curves. The phase differences $\Phi_{31}$ and $\Phi_{21}$ correlate for the RV Tauri light curves (red dots), showing that these sources form a homogeneous group of stars. The RV Tauri candidates (magenta open circles) also follow the relation that supports the RV Tauri nature of these stars. Furthermore we show the detected type II Cepheids as blue dots in this figure. The sequences of RV Tauri stars and type II Cepheids overlap, making the RV Tauri sequence an extension of the Cepheid sequence at higher periods. This supports the close connection between RV Tauri stars and type II Cepheids. In fact, Alcock et al. (1998) show that a single period-luminosity-color relationship describes both the type II Cepheids and RV Tauri stars in the LMC. We show the phase parameters for the stars presented in this study as green dots in Fig. 15. For this purpose we re-analyzed the light curves taken from the MACHO project database $\mathrm{e}^{3}$ and used the formal period to derive the Fourier parameters. Two stars with an uncertain classification were rejected from the analysis. The

\footnotetext{
${ }^{3}$ http://wwwmacho.mcmaster.ca/Data/MachoData.html
}

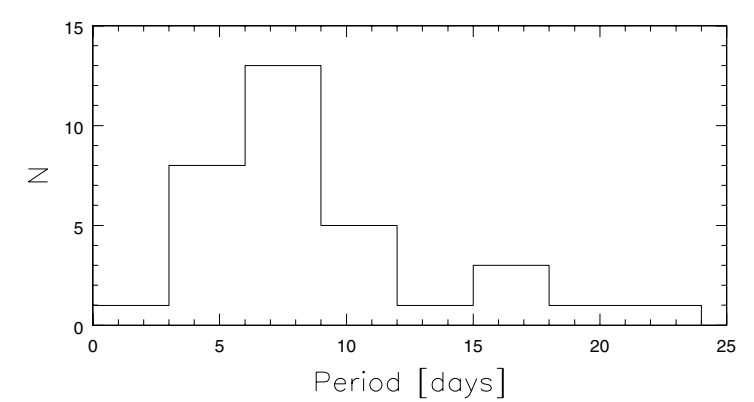

Fig. 16. Histogram of the periods of the Cepheid variables in group I.

LMC variables lie on the sequence of the WeCAPP RV Tauri and Cepheid II stars, supporting our classification of the variables in group II. However, as we are not able to resolve the RV Tauri stars due to the high surface brightness of the central parts of M 31 in the analyzed data set, we cannot finally prove the assignment of these stars as RV Tauri by placing the sources on a color-magnitude diagram.

The period range of the 93 type II Cepheids extends to periods longer than the approximate limit of about 20 days, as proposed by Alcock et al. (1998). There is still the possibility that some of these long-period Cepheids are in reality RV Tauri stars, since noise in the light curves can prevent us from detecting the alternation of deep and shallow maxima as required for assigning of the variable as a RV Tauri candidate or star. The majority of the light curves of this type of variable have a "flat-topped" shape (Kwee 1967) with relatively long and flat maxima. As already mentioned, the light curves of type II Cepheids show progression of the phase differences and occupy distinct places in Fourier space. We will discuss the Fourier parameters of the Cepheid-like variables in group I and II more thoroughly in a future publication. The sample of type II Cepheids outnumbers the sample of population I Cepheids by about a factor of three. As the type II Cepheids trace the old bulge population, this is not unexpected, even when taking their smaller brightness compared to population I Cepheids (at the same period) into account. The population I Cepheids, more massive and younger than the type II Cepheids, are typical members of the M31 disk population, which is superimposed on the M 31 bulge.

The third constituent of the group II sources are smallperiod semi-regular variables that contribute from periods of about 16 days onwards. The Fourier analysis of the light curves shows no correlations of the amplitude ratios or phase differences with period. Phase-phase diagrams also do not reveal a correlation of the phase parameters.

In Fig. 16 we give the period distribution of the population I Cepheids, whereas Fig. 17 shows the distribution of periods for variables belonging to group II. In red, magenta, blue, and green we present the distribution for the RV Tauri stars, RV Tauri candidates, type II Cepheids, and semi-regular variables.

\subsection{Group III - LPVs}

Group III consists of LPVs, i.e. Mira and semi-regular variable stars. LPVs are members of the AGB that marks the final stage 


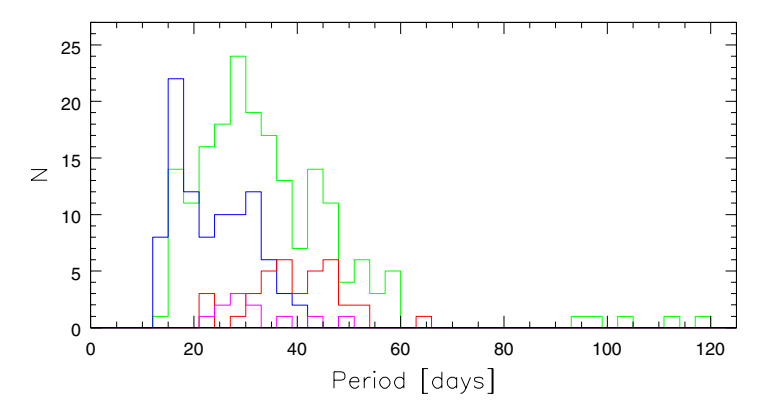

Fig. 17. Histogram of the periods of variables belonging to group II. We show the distributions for type II Cepheids (blue line), RV Tauri stars (red line), RV Tauri candidates (magenta line), and the semiregular stars (green line). For the RV Tauri stars and candidates, we use the single or fundamental period (minimum to minimum).

of the stellar evolution for intermediate mass stars with masses between 0.5 and $8 M_{\odot}$. These stars evolve from the main sequence and populate the red giant branch up to a maximum luminosity at the TRGB (tip of the red giant branch). After ignition of helium core burning, they drop in luminosity and form the horizontal branch. At the end of this stadium, the luminosity again rises as the stars evolve upwards the AGB. This evolution sequence is characterized by pulsation and extensive mass loss.

LPVs are a very promising tool for answering various astrophysical questions. They follow tight period-luminosity relations in the near-IR (especially the $K$-band) (Feast et al. 1989; Wood 2000; Feast et al. 2002), which makes them excellent galactic and extragalactic distance estimators. They are furthermore good indicators of the parent population to which they belong, as a dependence exists of the luminosity (hence, period) of Miras on the age. Longer period Miras should have higher mass progenitors and therefore belong to a younger population. LPVs are historically separated into two main groups: i) Miras with regular variations, periods between 80 and 1000 days, and an amplitude of the variation in the $V$-band of more than $2.5 \mathrm{mag}$; ii) semi-regulars (SR) with less regular variations, smaller periods, and a $V$-band variation smaller than 2.5 mag. Semi-regulars are divided in two groups, SRas with more regular variations and $\mathrm{SRbs}$ with less regular variations.

Recently Lebzelter et al. (2002) proposed a new classification scheme that is less dependent on this kind of artificial division and used it for the AGAPEROS survey of variable red stars towards the Magellanic Clouds. Their classification is based on the regularity of the variation alone, thereby providing three classes of stars: i) LPVs with regular variation; ii) LPVs with semi-regular variation; iii) LPVs with irregular variation. No cuts in amplitude were applied, therefore class i contains members of the classical Mira group, as well as members of the SRa type of objects.

We modified this classification scheme for our purposes and defined a significance cut of $P(>z)=10^{-20}$ according to the period-finding algorithm in $R$ or $I$ for the division of our sample of LPVs in classes $i$ (regular and semi-regular) and ii (irregular). Class ii coincides with the class of irregular stars introduced in Sect. 4. Because of the non-linear dependence of the significance on the $S / N$ of the light curve (see discussion below), we abandoned inventing an automatic cut for the

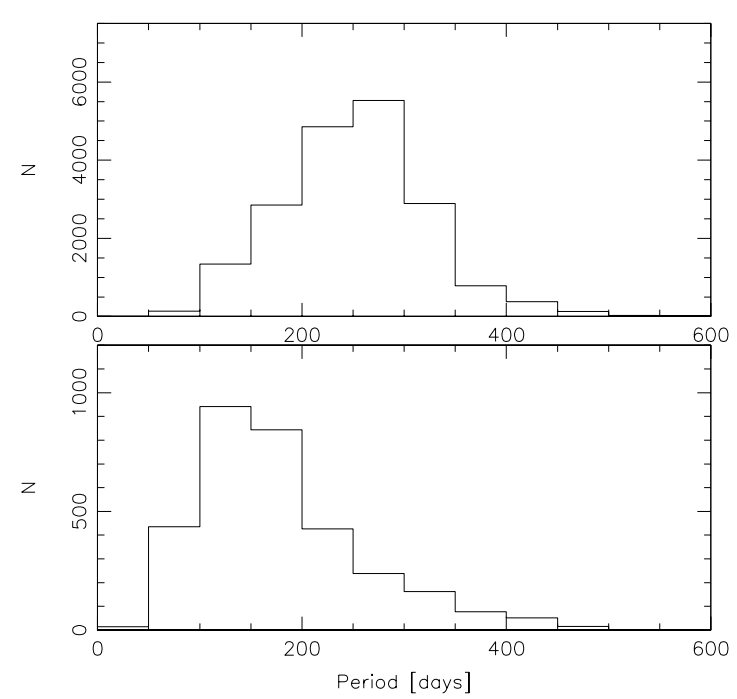

Fig. 18. Distribution of the periods of LPVs in the bulge of M 31 detected by the WeCAPP project. Top: class i - regular and semi-regular variables (significance level $P(>z)<10^{-20}$ in $R$ or I). Bottom: class ii - irregular variables (significance level $P(>z) \geq 10^{-20}$ in $R$ and $I$ ). Only irregulars selected by the significance cut are shown. Note that the distribution for the sources classified as irregular variables is of a qualitative nature only, as the periods for these sources are not welldetermined. In general irregular variables show smaller 'periods' than the group of regular and semi-regular variables.

division into semi-regular and regular variations. The cut at $10^{-20}$ is somewhat subjective, as stars with semi-regular variations, but low $S / N$ light curves are classified as irregular, since the low $S / N$ prevents the period being determined with better significance. On the other hand, this cut ensures that most of the irregular light curves are classified in the right way. The histograms of the periods for the irregular and the regular/semiregular sample of LPVs are shown in Fig. 18. The irregular variables show on average smaller "periods" than the group of regular or semi-regular variables. Note, that this statement is of qualitative nature only, as the periods for these sources are not well-determined.

We color-coded the LPVs in Fig. 12 according to the significance $P(>z)$ of the $I$-band period. The slope of the variation magnitude-period relation changes from slightly positive for the low significance tail (black dots) to negative for the sources with intermediate and high significant periods (green and red dots). The same trend can be seen in the left panel of Fig. 19, which shows the variation color $R-I$ as a function of the $R$-band variation magnitude. The significance of the $I$ band period rises for brighter $R$ variation magnitudes and redder variation colors. The differences in the significance of the LPV I-band periods, therefore, is a result of the different $S / N$ of the $I$-band light curves (rising $S / N$ in $I$ due to higher $R-I$ values), but can in part also be attributed to different stages of regularity in the light curves. The right panel of Fig. 19 finally shows the variation color $R-I$ as function of the period of the variable sources. The variation of the LPVs gets redder with increasing period, and at the same time shows more significant periods. 

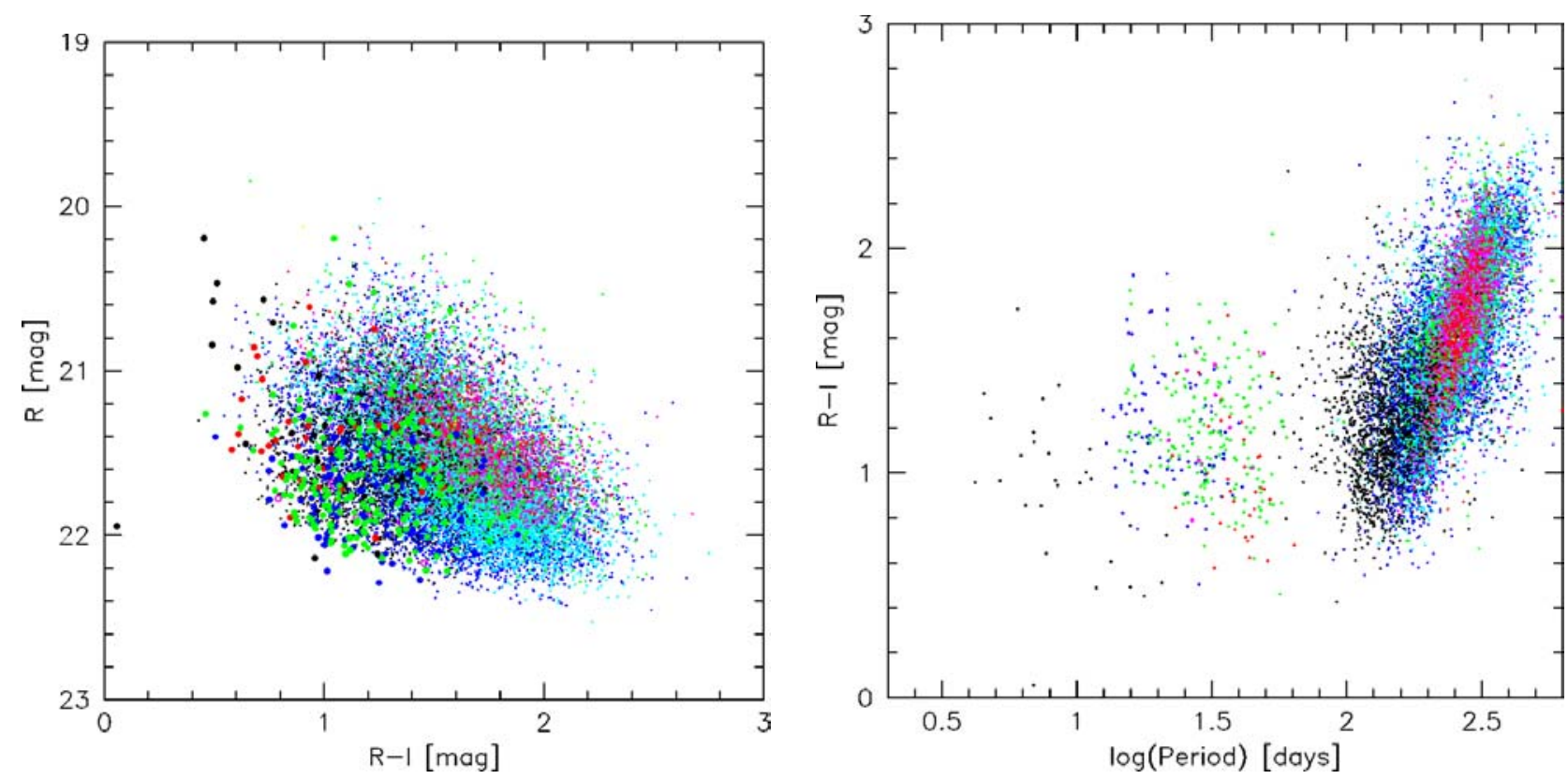

Fig. 19. Left panel: variation color $R-I$ shown as a function of the $R$-band variation magnitude. The sources of groups I and II are shown as big closed circles, the sources of group III as small closed circles. The color coding is the same as in Fig. 12, i.e. the LPVs are again color-coded according to the significance of the $I$-band period. The sources in groups I and II as a whole show bluer variations than the LPVs in group III. The significance of the $I$-band period rises for brighter $R$ variation magnitudes and redder variation colors. This is a result of the higher $S / N$ of the $I$-band light curves, but can in part also be attributed to the enhanced regularity of the light curves. Right panel: this figure shows the variation color $R-I$ as function of the period of the variable sources. Note that due to the normalization to the fluxes of Vega in the particular systems, this $R-I$ color should not be mistaken with the color of the variation amplitudes of the sources. Nevertheless, some trends are visible in this diagram: as the variation of the LPVs gets redder with increasing period, the $I$-band periods become more significant, reflecting the rising $S / N$ in $I$ due to higher $R-I$ values and a rising regularity of the light curves.

\section{The catalogue}

The final catalogue comprises 23781 entries with group I containing one beat Cepheid candidate, 2 s-Cepheids, one Cepheid of intermediate type, and 29 classical Cepheids pulsating in the fundamental mode. One further group I source, which remains unclassified in this catalogue, shows two periods.

Group II comprises 93 type II Cepheids, 37 RV Tauri stars, 11 RV Tauri candidates, and 193 low period semiregular variables. Group III consists of 4287 irregular and 18974 regular/semi-regular variables. The 82 presumably group III members show variations on longer timescales than the survey length. The positions on the $R$-band periodamplitude plane of the 31 eclipsing binary candidates coincide with the positions of groups I and II. Finally we detected 39 miscellaneous variables, among them 16 novae and $15 \mathrm{R}$ Coronae Borealis candidates.

The full catalog is available in electronic form at the CDS. We give the name, the position in the WCS, the periods (if available) derived from the $R$ - and $I$-band data, the amplitudes of the variation $\Delta F_{R}$ and $\Delta F_{I}$, and provide classification according to Sects. 4 and 5: DC (classical or $\delta$-Cepheids), SC (s-Cepheids), BC (beat Cepheids), IC (intermediate Cepheids), W (type II Cepheids), RV (RV Tauri stars), rv (RV Tauri candidates), S (regular or and semi-regular variations), and I (irregular variables). We also mark the identified Novae (N), eclipsing binary candidates (E), RCB candidates (RCB), and other miscellaneous variables (M). As an illustration of its contents, in
Table 2 we list the entries 100-120 in the catalogue. In Figs. 20 to 28 we show typical light curves for each of the groups.

\section{Correlation with other catalogues}

We cross-correlated our $R$-band selected catalogue with the General Catalogue of Variable Stars (GCVS, Durlevich et al. 1996) and catalogues selected in the $X$-ray (Kaaret 2002; Kong et al. 2002).

We found 23 coincidences with the Kaaret (2002) catalogue when using a search radius of 1 ". To estimate how many coincidences we expected by chance, we followed the approach proposed by Hornschemeier et al. (2001). We shifted one of the catalogues to be compared (in our case we shifted the catalogues taken from the literature) by $20^{\prime \prime}$ in north-east, southeast, south-west, and north-west directions, and checked for coincidences between these mock catalogues and our catalogue. By averaging these numbers, we got the expected number of coincidences by chance.

By applying a search radius of $1^{\prime \prime}$, the number of false coincidences with the Kaaret (2002) catalogue becomes 12 . This high level of false coincidences (about 50\%) results from the high number density of the variable star catalogue. Nevertheless, the false detections rate suggests that about 11 of the coincidences should be real. This is supported by the fact that 6 of the coincident sources have an entry in the 2 MASS catalogue (Cutri et al. 2000), and 8 of the KaaretWeCAPP sources are identified as globular clusters (4 of them 

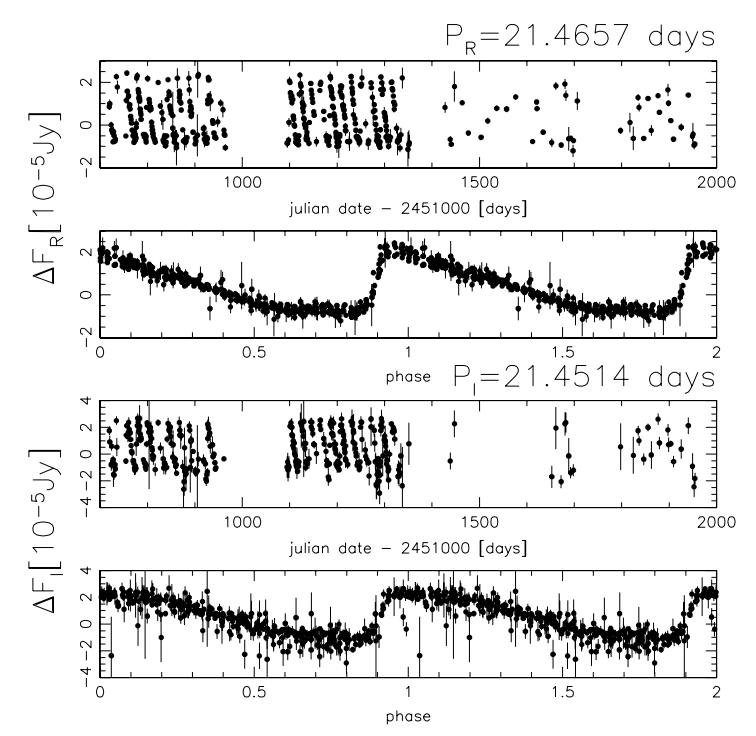

Fig. 20. Light curve of a $\delta$-Cepheid (group I). In the upper panels we show the light curve $R$-band, while the lower panels show the light curves in the $I$-band. In both bands the light curves are presented in the time domain (top for each band) and in the phase domain, i.e. convolved with the derived periods (bottom for each band). We also show the periods $P_{R}$ and $P_{I}$ derived in the $R$ - and $I$-band, respectively.

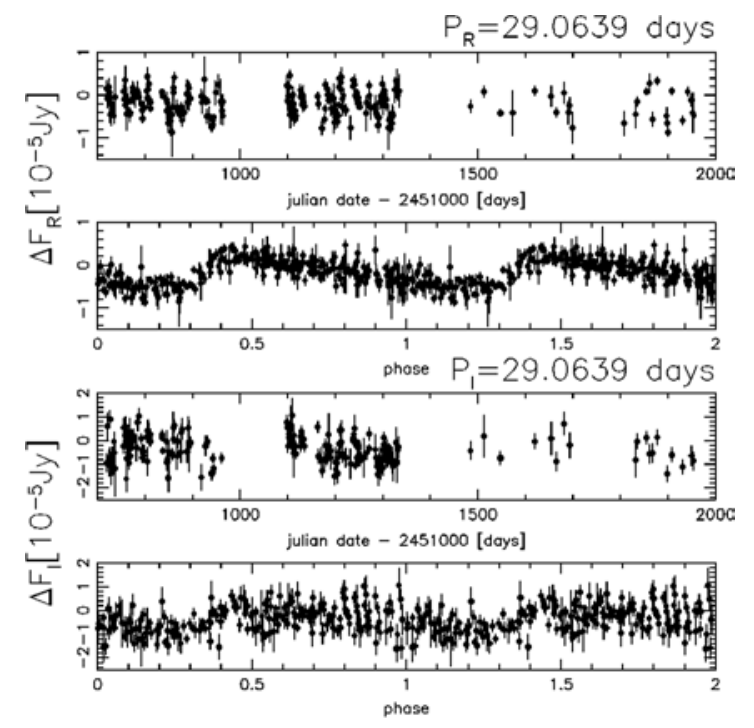

Fig. 21. Light curve of a long period type II Cepheid (group II) in the $R$-band (top panels) and the I-band (bottom panels).

with a 2MASS entry, Kaaret 2002). Furthermore, we found that 13 of the sources have coincident counterparts in the Kong et al. (2002) catalogue as well (see below). In Table 3 we give the positions of all 23 coincidences, and we give the 2MASS name also for sources with entries in the 2MASS catalogue. Two of the Kaaret-WeCAPP sources with 2MASS fluxes (WeCAPP_V8946 and WeCAPP_V10578) have no globular cluster (gc) counterpart Kaaret (2002). While V8946 coincides with a gc-candidate identified by Wirth, Smarr, \& Bruno (1985), a gc-counterpart for WeCAPP_V10578 is completely unknown. Another two of the WeCAPP-Kaaret sources (V16322 and V10431) have been identified as optical novae showing supersoft X-ray emission (Pietsch et al. 2005).

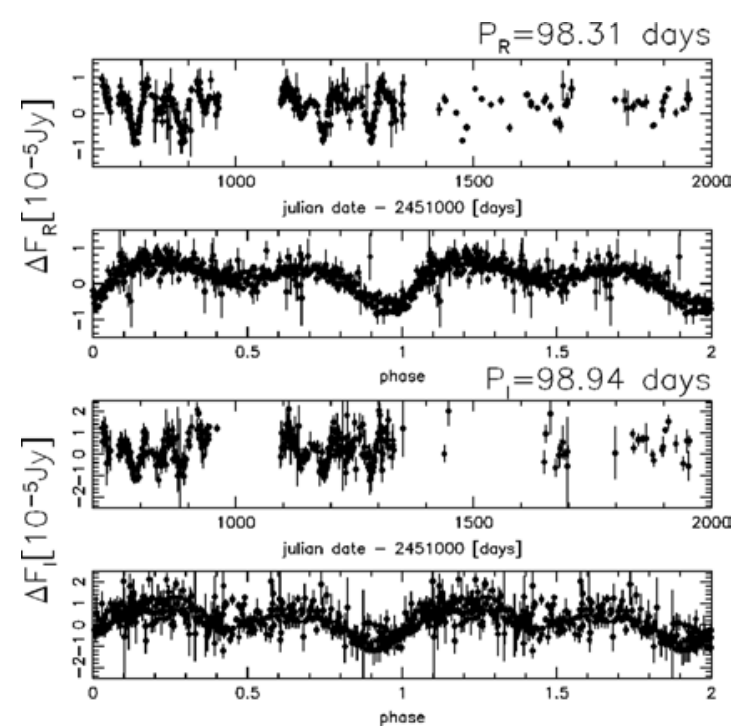

Fig. 22. Light curve of a RV Tauri star (group II) in the $R$-band (top panels) and the I-band (bottom panels). We show the light curves convolved with the formal periods (deep minimum to deep minimum). The double wave light curve with alternating deep and shallow minima is nicely uncovered.

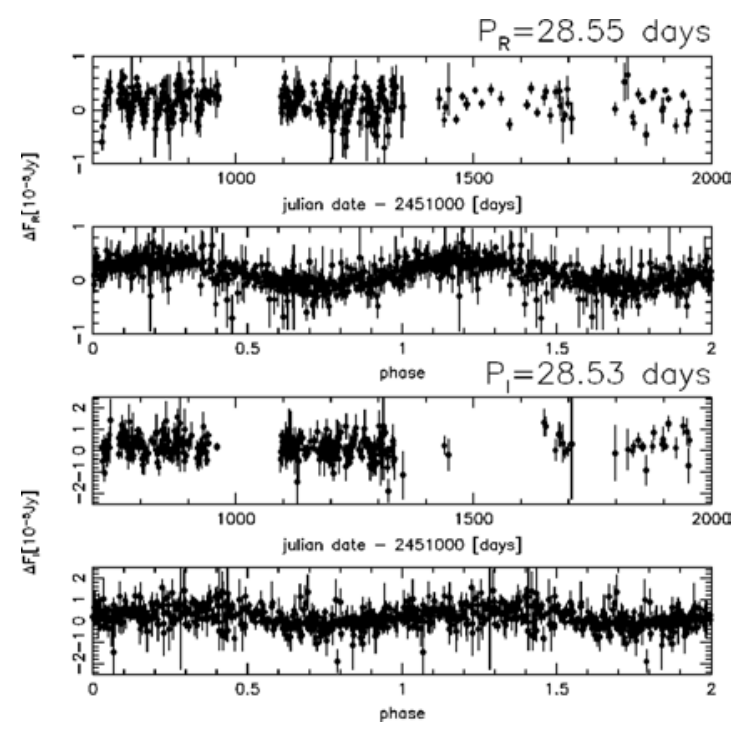

Fig. 23. Light curve of a low period semi-regular variable (group II) in the $R$-band (top panels) and the I-band (bottom panels).

The Kong et al. (2002) catalogue contains 31 coincidences with our variable star catalogue when applying a 1 " search radius. As we expect 17 coincidences by chance, about 14 of the coincidences should be real. As mentioned above, 13 of the sources are also coincident with the Kaaret (2002) catalogue. According to Kong et al. (2002), 12 of the sources have a globular cluster counterpart ( 3 " search radius), and 22 of the coincidences are variable in the X-rays at the $3-\sigma$ level. We show the positions and identifiers of all 31 coincidences in Table 4.

The GCVS (Durlevich et al. 1996) comprises 250 entries within our field of view, most of them of nova type. Of there 161 (6) were classified as novae, along with 53 as novae of fast type (NA), 9 (2) of slow type (NB), and (2) of very slow type (NC). Furthermore the GCVS reports 1 SNIa remnant in 


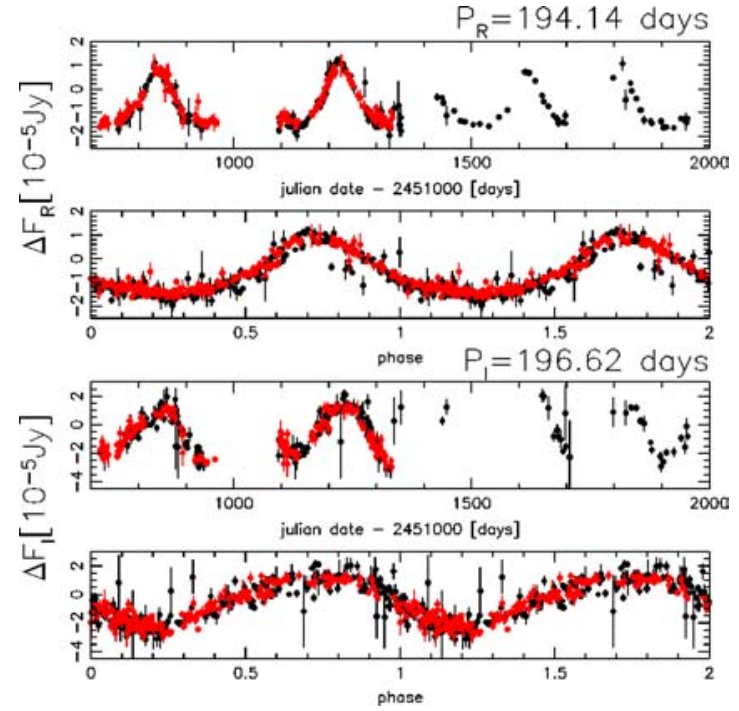

Fig. 24. Light curve of an LPV with regular variation (group III) in the $R$-band (top panels) and the $I$-band (bottom panels). As illustration of the agreement of the data taken with different telescopes, we show the data collected at Calar Alto as grey dots (red dots in the online version) and data taken at Wendelstein as black dots.

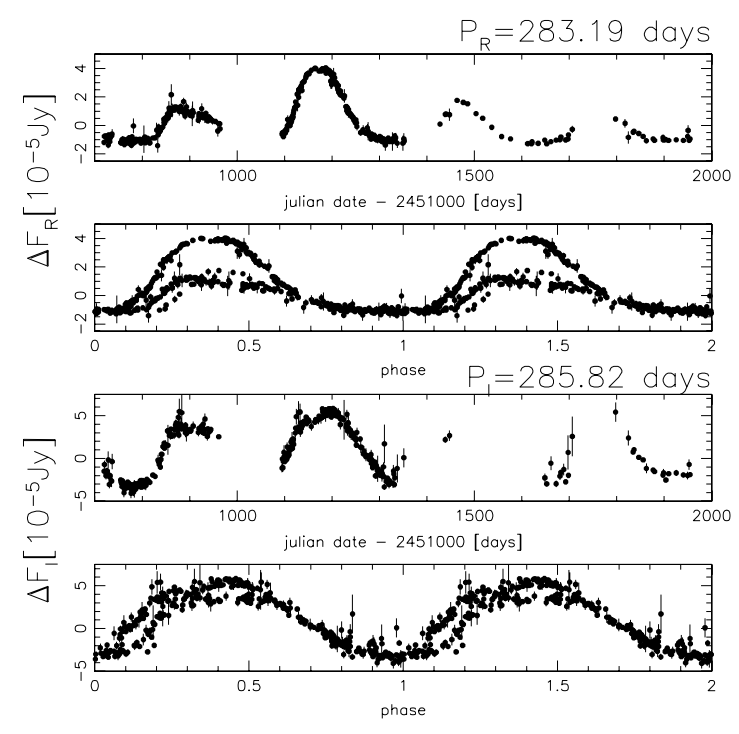

Fig. 25. Light curve of a LPV (group III) with changing variation amplitudes from cycle to cycle. Top panels: $R$-band. Bottom panels: I-band.

our field, 1 (1) LC type (irregular variable supergiant), 3 (1) semi-regular variables (SR), $3 \delta$-Cepheids, (1) SDOR variable, $2(1+2)$ irregular variables, and 1 unstudied (S: classification) variable. The numbers in brackets give additional candidates for the particular variable star group with questionable classifications according to the GCVS.

The crowding of the WeCAPP sources demands that we keep the search radius at $1^{\prime \prime}$, even when comparing with the GCVS, whose accuracy is well below this value. We found 27 coincidences; the number of false detections (21) suggests, however, that most of the coincidences are not real. As 23 of them are classified as novae in the GCVS, we only show the 4 coincidences that we regard as real in Table 5. Note, that we
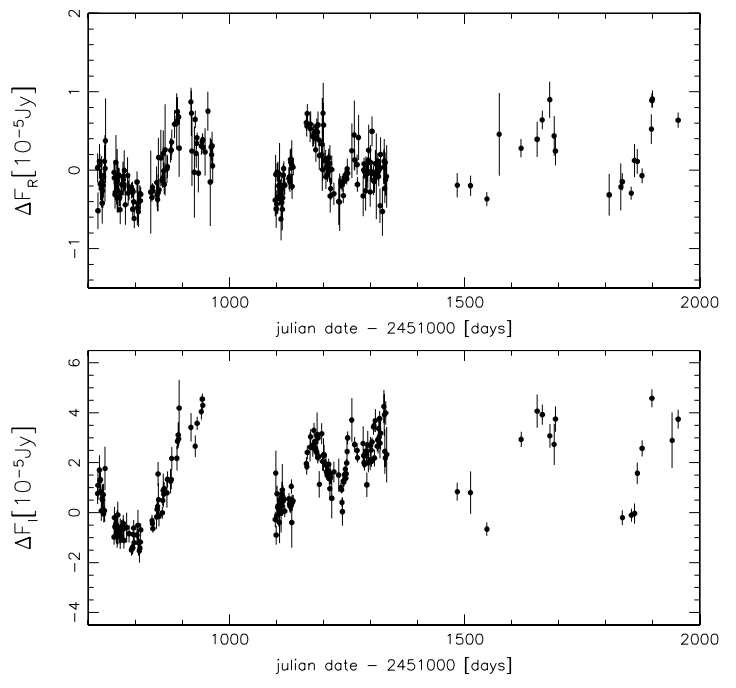

Fig. 26. Light curve of an irregular LPV (group III). Top panel: $R$-band. Bottom panel: I-band.
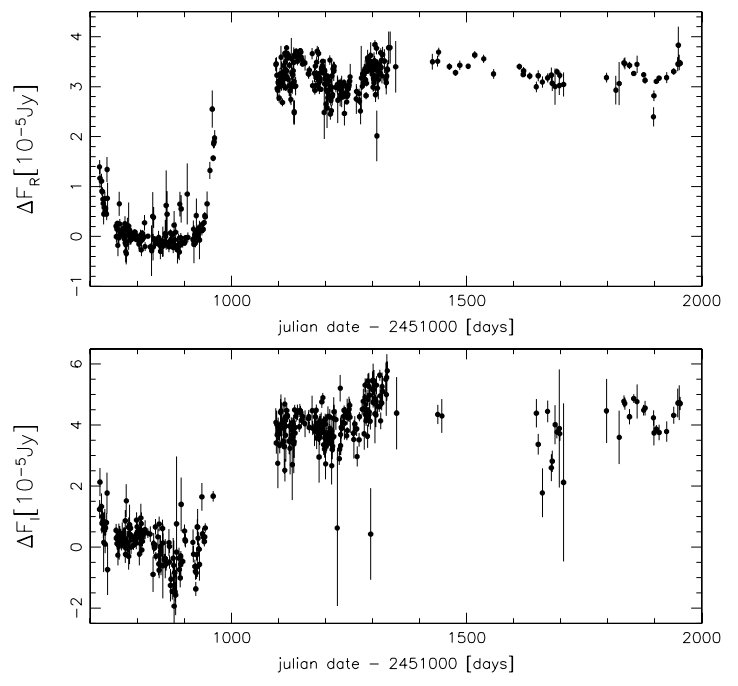

Fig. 27. Light curve of a R Coronae Borealis candidate (miscellaneous variables). Top panel: $R$-band. Bottom panel: I-band. These rare carbon-rich stars show unpredictable and frequent fading of light in optical wavebands, most probably due to dust grains ejected from the stellar photosphere. For a review of these RCB stars see Clayton (1996).

do not match the positions of all three $\delta$-Cepheids found in the GCVS inside our field. The GCVS Cepheid V0934 has a position difference of 2.1" when compared to our position, and GCVS Cepheid V0811 is off by $10.2^{\prime \prime}$. Because of these in part large uncertainties in the positions of the GCVS, we are not trying to match the two remaining semi-regulars, given their smaller variation amplitude.

\section{Conclusions and outlook}

The WeCAPP observations in the optical $R$ and $I$ bands towards the bulge of M 31 covering the years 2000-2003 with very good time sampling resulted in a database of over 23000 variable sources. In this paper we have presented the catalogue of variable stars identified in the central parts of M 31. 

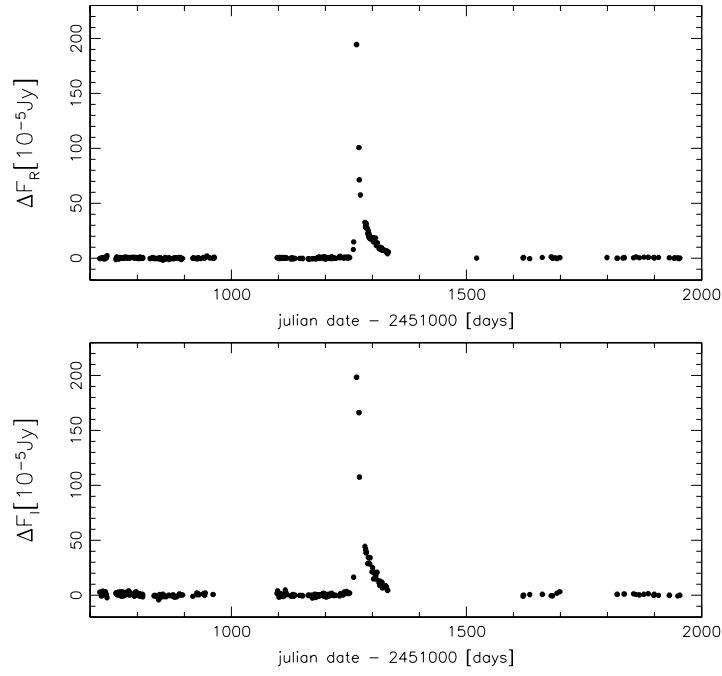

Fig. 28. Light curve of a nova (miscellaneous variables). Top panel: $R$-band. Bottom panel: $I$-band. A paper concerning the characteristics of all detected novae is in preparation.

The distribution of the detected variable sources shows an asymmetry due to the enhanced extinction in the spiral arms projected on the M 31 bulge. Assuming that the non-variable stars in the bulge behave in the same way as the variable counterparts, this asymmetry enlarges the expected asymmetry in the microlensing signal resulting from the high inclination of M 31. Theoretical calculations, therefore, have to take a modified distribution of sources (or the distribution of the extinction present in a given band) into account.

The catalogue containing regular, semi-regular and irregular LPVs, Cepheids, RV Tauri stars, eclipsing binary, and R Coronae Borealis candidates enlightens the rich population of variable sources in M 31, which can then be exploited for various astrophysical questions. Two more papers dealing with the Fourier parameters for the Cepheid-like stars and the WeCAPP nova catalogue are in preparation. A fraction of the nova catalogue has already been correlated with supersoft X-ray sources (SSSs) in M 31, showing that classical novae constitute the major class of SSSs in M 31 (Pietsch et al. 2005).

Recently it was proposed that sequence E, first detected by Wood (1999) in the MACHO data towards the LMC bar, is populated by ellipsoidal red giants (Soszynski et al. 2004). Wood (1999) found five distinct period-luminosity sequences, of which three could be attributed to different evolutionary phases and pulsation modes. For two of them, sequences D and E, it was suggested that they are populated by contact binaries and by semi-detached binaries with an invisible companion. Analyzing the data of the LMC ellipsoidal candidates from the OGLE web archive ${ }^{4}$ and accounting for the distance modulus of M 31 proves that these sources are below the detection limit of our survey. Therefore, we cannot put any constraints on a possible population of ellipsoidal red giants in M 31. The more detailed examination of the group III LPVs will be subject to future work. Until now we have measured the dominant period

\footnotetext{
${ }^{4}$ http://ogle.astrouw.edu.pl/
}

in these stars that are known to show multi-periodic phenomena. Future work will include extraction of the sub-dominant periods and distribution of the ratio of the different periods. Furthermore, the distribution and possible correlations of the Fourier parameters for these stars is an interesting and, until now, unexamined problem which we can address with this data set.

Acknowledgements. The authors thank the referee Jean-Philippe Beaulieu for his comments, which improved the manuscript considerably. We would like to thank the observers and staff at Wendelstein Observatory, Otto Bärnbantner, Christoph Riess, Heinz Barwig, Claus Gössl, and Wolfgang Mitsch, and all the staff at Calar Alto Observatory for their extensive support during the observing runs of this project. JF thanks Armin Gabasch and Ulrich Hopp for stimulating discussions. This work was supported by the Sonderforschungsbereich 375-95 Astro-Particle-Physics of the Deutsche Forschungsgemeinschaft.

\section{References}

Alard, C., \& Lupton, R. 1998, ApJ, 503, 325

Alard, C. 1999, A\&A, 343, 10

Alard, C. 2000, A\&AS, 144, 363

Alcock, C., Allsman, R. A., Axelrod, T. S., et al. 1995, AJ, 109, 1653

Alcock, C., Allsman, R. A., Alves, D. R., et al. 1998, AJ, 115, 1921

Alcock, C., Allsman, R. A., Axelrod, T. S., et al. 1999, ApJ, 511, 185 An, J. H., Evans, N. W., Hewett, P., et al. 2004, MNRAS, 351, 1071

Ansari, R., Auriere, M., Baillon, P., et al. 1997, A\&A, 324, 843

Ansari, R., Auriere, M., Baillon, P., et al. 2004, A\&A, 421, 509

Antonello, E., \& Poretti, E. 1986, A\&A, 169, 149

Auriere, M., Baillon, P., Bouquet, A., et al. 2001, ApJ, 553, 137

Baade, W., \& Swope, H. H. 1963, AJ, 68, 435

Baade, W., \& Swope, H. H. 1965, AJ, 70, 212

Beaulieu, J. P., Grison, P., Tobin, W., et al. 1995, A\&A, 303, 137

Beaulieu, J. P., Krockenberger, M., Sasselov, D. D., et al. 1997, A\&A, 321, L5

Bessell, M. S. 1979, PASP, 91, 589

Bertin, E., \& Arnouts, S. 1996, A\&AS, 117, 393

Binney, J., \& Merrifield, M. 1988, in Galactic Astronomy, Princeton Series in Astrophysics

Baillon, P., Bouquet, A., Giraud-Heraud, Y., \& Kaplan, J. 1993, A\&A, 277,1

Calchi Novati, S., Iovane, G., Marino, A. A. et al. 2002, A\&A, 381, 848

Cassisi, S., Castellani, V., degl'Innocenti, S., \& Weiss, A. 1998, A\&AS, 129, 267

Cieslinski, D., Diaz, M. P., Mennickent, R. E., \& Pietrzynski, G. 2003, PASP, 115, 193

Clayton, G. C. 1996, PASP, 108, 225

Crotts, A. P. S. 1992, ApJ, 399, 43

Cutri, R. M., et al., Explanatory Supplement to the 2MASS Second Incremental Data Release

de Jong, J. T. A., Kuijken, K., Crotts, A. P. S., et al. 2004, A\&A, 417, 461

Derue, F., Marquette, J.-B., Lupone, S., et al. 2002, A\&A, 389, 149

Durlevich, O., Kazarovets, E., Kholopov, P., et al. 1996, in The General Catalogue of Variable Stars, fourth edition, as available from the CDS

Feast, M. W., Glass, I. S., Whitelock P., \& Catchpole, R. M. 1989, MNRAS, 241, 375

Feast, M. W., Whitelock, P., \& Menzies, J. 2002, MNRAS, 329, L7 
Fokin, A. B. 2001, in Stellar Pulsation-Nonlinear Studies, ed. M., Takeuti, \& D. D., Sasselov (Kluwer Academic Publishers), 103

Gössl, C. A., \& Riffeser, A. 2002, A\&A, 381, 1095

Grillmair, C. J., Lauer, T. R., Worthey, G., et al. 1996, AJ, 112, 1975

Haiman, Z., Magnier, E., Lewin, W. H. G., et al. 1994, A\&A, 286, 725

Hertzsprung, E. 1924, BAN, 3, 115

Horne, J. H., \& Baliunas, S. L. 1986, ApJ, 302, 757

Hornschemeier, A. E., Brandt, W. N., Garmire, G. P., et al. 2001, ApJ, 554,742

Hubble, E. P. 1922, ApJ 56, 162

Iben, I., \& Renzini, A. 1983, ARA\&A, 21, 271

Jablonka, P., Bridges, T. J., Sarajedini, A., et al. 1999, ApJ, 518, 627

Kaaret, P. 2002, ApJ, 578, 114

Kaluzny, J., Stanek, K. Z., Krockenberger, M., et al. 1998, AJ, 115,1016

Kent, S. 1989, AJ, 97, 1614

Kong, A.K.H., Garcia, M.R., Primini F.A. et al. 2002, ApJ, 577, 738

Kovacs, G., Zucker, S., \& Mazeh, T. 2002, A\&A, 391, 369

Kwee, K. K., Bull. Astron. Inst. Netherlands, 19, 260

Lebzelter T., Schultheis M., \& Melchior A.L. 2002, A\&A, 393, 573

Lomb, N. R. 1976, Ap\&SS, 39, 447

Magnier, E. A., Lewin, W. H. G., van Paradijs, J. et al. 1992, A\&AS, 96, 379

Noda, S., Takeuti, M., Abe, F., et al. 2002, MNRAS, 330, 137

Noda, S., Takeuti, M., Abe, F. et al. 2004, MNRAS, 348, 1120

Maraston, C. 1998, MNRAS, 300, 872

Massey, P., Hodge, P. W., Holmes, S., et al. 2002, A\&AS, 199th meeting, Washington, DC, 130.05
Pietsch, W., Fliri, J., Freyberg, M. J., et al., 2005, A\&A, 442, 879

Press W. H., Teukolsky S. A., Vetterling, W. T., \& Flannery, B. P. 1988, Numerical Recipes (Cambridge University Press)

Rejkuba, M., Minniti, D., Silva, D. R., \& Bedding, T. R. 2003, A\&A, 411, 351

Renzini, A. 1998, AJ, 115, 2459

Riffeser, A., Fliri, J., Gössl, C. A., et al. 2001, A\&A, 379, 362

Riffeser, A., Fliri, J., Bender, R., Seitz, S., Gössl, C. A. 2003, ApJ, 599, L17

Riffeser, A., et al., in prep.

Roberts D. H., Lehar J., \& Dreher J. W. 1987, AJ, 93, 968

Scargle, J. D. 1982, ApJ, 263, 835

Simon, N. R., \& Lee, A. 1981, ApJ, 248, 291

Simon, N. R., \& Moffett, T. J. 1986, PASP, 97, 1078

Stanek, K. Z., \& Garnavich, P. M. 1998, ApJ, 503, 131

Soszynski, I., Udalski, A., Kubiak, M., et al. 2004, Acta, 54, 347

Szymanski, M., Kubiak, M., \& Udalski, A. 2001, Acta, 51, 259

Stanek, K. Z., \& Garnavich, P. M. 1998, ApJ, 503, 131

Tingley, B. 2003, A\&A, 408, L5

Tomaney, A. B., \& Crotts, A. P. S. 1996, AJ, 112, 2872

Wallerstein, G. 2002, PASP, 111, 689

Whitelock, P., Marang, F., \& Feast, M. 2000, MNRAS, 319, 728

Wirth, A., Smarr, L. L., \& Bruno, T. L. 1985, ApJ, 290, 140

Wood, P. R. 1999, IAUS, 191, 151

Wood, P. R. 2000, PASA, 17, 18

Wray, J. J., Eyer, L., \& Paczynski B. 2004, MNRAS, 349, 1059 